\title{
Reliable Theoretical Procedures for Calculating the Rate of Methyl Radical Addition to Carbon-Carbon Double and Triple Bonds
}

\author{
Rodolfo Gómez-Balderas, ${ }^{\dagger, \neq, \mathbb{l}}$ Michelle L. Coote, ${ }^{\ddagger} *$ David J. Henry, ${ }^{\ddagger}$ and Leo \\ $\operatorname{Radom}^{\ddagger}$,II** \\ Research School of Chemistry, Australian National University, Canberra, ACT 0200, \\ Australia, and School of Chemistry, University of Sydney, Sydney, NSW 2006, Australia
}

\section{SUPPORTING INFORMATION}

(Tables S1-S8, Total 23 pages)

\footnotetext{
${ }^{\dagger}$ On postdoctoral leave from FESC-UNAM (México)

* Australian National University

"University of Sydney

*To whom correspondence should be addressed, email: mcoote@ rsc.anu.edu.au; radom@chem.usyd.edu.au
} 


\title{
TABLE S1: GAUSSIAN Archive Entries for the Optimized Geometries $\cdot \mathrm{CH}_{3}$
}

\author{
$\mathrm{UHF} / 6-31 \mathrm{G}(\mathrm{d})$
}

$1 \backslash 1 \backslash G I N C-S C 61 \backslash F O p t \backslash U H F \backslash 6-31 G(d) \backslash C 1 H 3(2) \backslash R G B 501 \backslash 22-A u g-2002 \backslash 0 \backslash \backslash \# N$ UHF 6

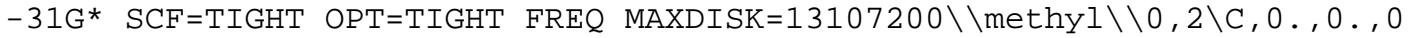
$. \backslash \mathrm{H}, 1.0725674901,0 ., 0 . \backslash \mathrm{H},-0.536283745,-0.9288706937,0 . \backslash \mathrm{H},-0.536283745$, $0.9288706937,0 . \backslash \backslash$ Version=DEC-AXP-OSF /1-G98RevA.11.3 $\backslash \mathrm{HF}=-39.558992 \backslash \mathrm{S} 2=0$ $.761485 \backslash S 2-1=0 . \backslash S 2 A=0.750074 \backslash R M S D=3.697 e-09 \backslash R M S F=1.250 e-09 \backslash D i p o l e=0 ., 0$ ., $0 . \backslash \mathrm{PG}=\mathrm{D} 03 \mathrm{H} \quad[\mathrm{O}(\mathrm{C} 1), 3 \mathrm{C} 2(\mathrm{H} 1)] \backslash \backslash \mathrm{Q}$

\section{UB3LYP / 6-31G (d)}

$1 \backslash 1 \backslash G I N C-S C 116 \backslash F O p t \backslash U B 3 L Y P \backslash 6-31 G(d) \backslash C 1 H 3(2) \backslash R G B 501 \backslash 22-A u g-2002 \backslash 0 \backslash \backslash \# N B$ 3LYP 6-31G(D) SCF=TIGHT OPT=TIGHT FREQ MAXDISK=13107200 \methyl Opt\&Er

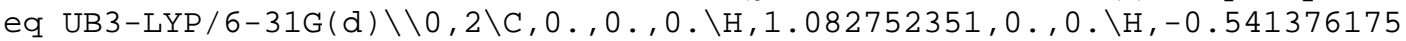
$5,-0.937691042,0 . \backslash \mathrm{H},-0.5413761755,0.937691042,0 . \backslash \backslash$ Version=DEC-AXP $-O S E /$ $1-G 98 R e v A .11 .3 \backslash H F=-39.8382922 \backslash S 2=0.753765 \backslash S 2-1=0 . \backslash S 2 A=0.750007 \backslash R M S D=4$. $410 \mathrm{e}-09 \backslash \mathrm{RMSF}=1.426 \mathrm{e}-09 \backslash \mathrm{Dipole}=0 ., 0 ., 0 . \backslash \mathrm{PG}=\mathrm{D} 03 \mathrm{H} \quad[\mathrm{O}(\mathrm{C} 1), 3 \mathrm{C} 2(\mathrm{H} 1)] \backslash \backslash \mathrm{e}$

\section{UB3LYP / 6-31G (2df, p)}

$1 \backslash 1 \backslash G I N C-S C 8 \backslash F O p t \backslash U B 3 L Y P \backslash 6-31 \mathrm{G}(2 \mathrm{df}, \mathrm{p}) \backslash \mathrm{C} 1 \mathrm{H} 3(2) \backslash R G B 501 \backslash 05-M a y-2002 \backslash 0 \backslash \backslash \# N$ UB3LYP 6-31G (2DF, P) SCF=(DIRECT, TIGHT) OPT FREQ MAXDISK=117964800 \me thyl Opt\&Freq UB3-LYP/6-31G $(2 \mathrm{df}, \mathrm{p}) \backslash \backslash 0,2 \backslash \mathrm{C}, 0 ., 0 ., 0 . \backslash \mathrm{H}, 1.081363225,0 ., 0$. $\backslash \mathrm{H},-0.5406816125,-0.9364880236,0 . \backslash \mathrm{H},-0.5406816125,0.9364880236,0 . \backslash \backslash$ Ver sion=DEC-AXP $-O S F / 1-G 98 R e v A .11 .3 \backslash \mathrm{HF}=-39.8433562 \backslash \mathrm{S} 2=0.753952 \backslash \mathrm{S} 2-1=0 . \backslash \mathrm{S} 2 \mathrm{~A}$ $=0.750008 \backslash \mathrm{RMSD}=8.817 \mathrm{e}-10 \backslash \mathrm{RMSF}=1.719 \mathrm{e}-05 \backslash \mathrm{Dipole}=0,, 0 ., 0 . \backslash \mathrm{PG}=\mathrm{D} 03 \mathrm{H} \quad[\mathrm{O}(\mathrm{C} 1)$ , 3C2 ( $\mathrm{H} 1)] \backslash \backslash \mathrm{C}$

\section{UB3LYP/CC-PVTZ}

$1 \backslash 1 \backslash G I N C-S C 35 \backslash F O p t \backslash U B 3 L Y P \backslash C C-p V T Z \backslash C 1 H 3(2) \backslash R G B 501 \backslash 10-A p r-2002 \backslash 1 \backslash \backslash \# N$ B3L YP CC-PVTZ SCF=DIRECT OPT=(Z-MATRIX, TIGHT) FREQ MAXDISK=13107200\\meth

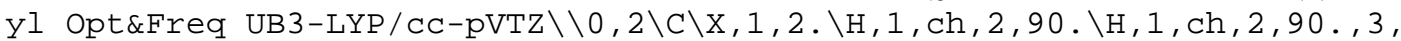
$120 ., 0 \backslash \mathrm{H}, 1, \mathrm{ch}, 2,90 ., 3,-120 ., 0 \backslash \backslash \mathrm{ch}=1.07801147 \backslash \backslash$ Version=DEC-AXP-OSF /1-G 9 8RevA.11.3 State=2-A2 $\backslash \mathrm{HF}=-39.8586717 \backslash \mathrm{S} 2=0.753617 \backslash \mathrm{S} 2-1=0 . \backslash \mathrm{S} 2 \mathrm{~A}=0.750007$ $\backslash \mathrm{RMSD}=8.086 \mathrm{e}-09 \backslash \mathrm{RMSF}=4.735 \mathrm{e}-09 \backslash \mathrm{Dipole}=0 ., 0 ., 0 . \backslash \mathrm{PG}=\mathrm{D} 03 \mathrm{H} \quad[\mathrm{O}(\mathrm{C} 1), 3 \mathrm{C} 2(\mathrm{H} 1)]$ $\backslash \backslash Q$

\section{UB3LYP / 6-311+G (3df, 2p)}

$1 \backslash 1 \backslash G I N C-S C 14 \backslash F O p t \backslash U B 3 L Y P \backslash 6-311+G(3 d f, 2 p) \backslash C 1 H 3$ (2) RGB501\22-Aug-2002\0 $\backslash \backslash \# N$ B3LYP $6-311+G(3 D F, 2 P) \quad S C F=T I G H T$ OPT=TIGHT FREQ MAXDISK=13107200 \

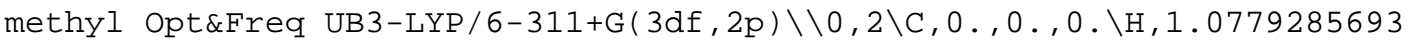
$, 0 ., 0 . \backslash \mathrm{H},-0.5389642846,-0.9335135244,0 . \backslash \mathrm{H},-0.5389642846,0.9335135244,0$ . $\backslash \backslash$ Version=DEC-AXP-OSF /1-G98RevA.11.3 State=2-A2" $\backslash H F=-39.8577854 \backslash S 2=0$. $753566 \backslash S 2-1=0 . \backslash S 2 A=0.750006 \backslash R M S D=6.741 e-09 \backslash R M S F=3.948 e-07 \backslash D i p o l e=0 ., 0$. , $0 . \backslash \mathrm{PG}=\mathrm{D} 03 \mathrm{H} \quad[\mathrm{O}(\mathrm{C} 1), 3 \mathrm{C} 2(\mathrm{H} 1)] \backslash \backslash \mathrm{C}$

\section{UMPW1K/6-31+G (d,p)}

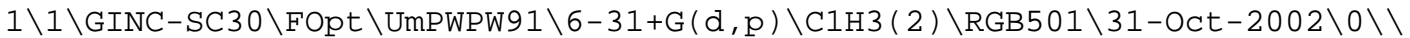
\#MPWPW91 6-31+G** IOP (5/45=10000428) IOP (5/46=05720572) IOP (5/47=10001

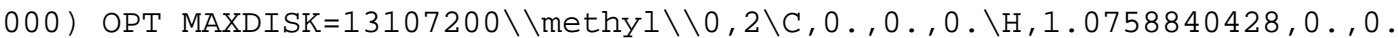
$\backslash \mathrm{H},-0.5379420214,-0.9317429126,0 . \backslash \mathrm{H},-0.5379420214,0.9317429126,0 . \backslash \backslash \mathrm{Ver}$ sion=DEC-AXP -OSF $/ 1-G 98 R e v A .11 .3 \backslash \mathrm{HF}=-39.8347286 \backslash \mathrm{S} 2=0.754803 \backslash \mathrm{S} 2-1=0 . \backslash \mathrm{S} 2 \mathrm{~A}$ $=0.750011 \backslash \mathrm{RMSD}=8.700 \mathrm{e}-10 \backslash \mathrm{RMSF}=1.091 \mathrm{e}-04 \backslash \mathrm{Dipole}=0,0 ., 0 . \backslash \mathrm{PG}=\mathrm{D} 03 \mathrm{H} \quad[\mathrm{O}(\mathrm{C} 1)$ , 3C2 (H1) ] \\@

\section{UMP2 /6-31G (d)}

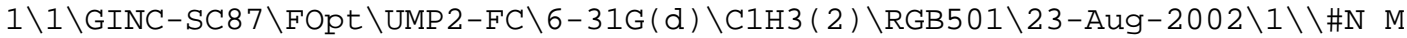
P2 6-31G* SCF=TIGHT OPT $=($ TIGHT, Z-MATRIX) FREQ MAXDISK=13107200 \methyl $\mathrm{UMP} 2 \backslash \backslash 0,2 \backslash \mathrm{C} \backslash \mathrm{X}, 1,2 . \backslash \mathrm{H}, 1, \mathrm{ch}, 2,90 . \backslash \mathrm{H}, 1, \mathrm{ch}, 2,90 ., 3,120.0 \backslash \mathrm{H}, 1, \mathrm{ch}, 2,90 ., 3$, 
$-120 ., 0 \backslash \backslash \mathrm{Ch}=1.07873864 \backslash \backslash$ Version=DEC-AXP-OSF $/ 1-G 98 \mathrm{RevA} .11 .3 \backslash \mathrm{HF}=-39.5589$ $09 \backslash \mathrm{MP} 2=-39.6687503 \backslash \mathrm{PUHF}=-39.5619922 \backslash \mathrm{PMP} 2-0=-39.6707475 \backslash \mathrm{S} 2=0.761796 \backslash \mathrm{S} 2-$ $1=0.753452 \backslash \mathrm{S} 2 \mathrm{~A}=0.750079 \backslash \mathrm{RMSD}=3.371 \mathrm{e}-09 \backslash \mathrm{RMSF}=4.372 \mathrm{e}-10 \backslash \mathrm{Dipole}=0 ., 0 ., 0 . \backslash$ $\mathrm{PG}=\mathrm{D} 03 \mathrm{H} \quad[\mathrm{O}(\mathrm{C} 1), 3 \mathrm{C} 2(\mathrm{H} 1)] \backslash \backslash \mathrm{C}$

\section{UQCISD/6-31G (d)}

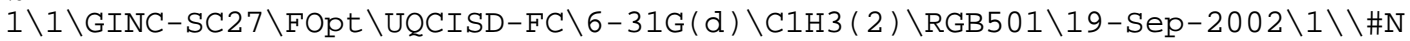
QCISD 6-31G* FOPT (TIGHT, Z-MATRIX) FREQ MAXDISK=104857600 \methyl \\0,2 $\backslash \mathrm{C} \backslash \mathrm{X}, 1,2 . \backslash \mathrm{H}, 1, \mathrm{ch}, 2,90 . \backslash \mathrm{H}, 1, \mathrm{ch}, 2,90 ., 3,120.0 \backslash \mathrm{H}, 1, \mathrm{ch}, 2,90 ., 3,-120 ., 0 \backslash \backslash \mathrm{C}$ $\mathrm{h}=1.08359853 \backslash \backslash$ Version=DEC-AXP-OSF $/ 1-G 98$ RevA. $11.3 \backslash \mathrm{HF}=-39.5587292 \backslash \mathrm{MP} 2=-3$ $9.6687008 \backslash \mathrm{MP} 3=-39.6846254 \backslash \mathrm{MP} 4 \mathrm{D}=-39.6884836 \backslash \mathrm{MP} 4 \mathrm{DQ}=-39.68716 \backslash \mathrm{PUHF}=-39.56$ $18424 \backslash \mathrm{PMP} 2-0=-39.6707259 \backslash \mathrm{PMP} 3-0=-39.6858615 \backslash \mathrm{MP} 4 \mathrm{SDQ}=-39.68778 \backslash \mathrm{QCISD}=-39$ $.6891219 \backslash \mathrm{S} 2=0.762048 \backslash \mathrm{S} 2-1=0.753591 \backslash \mathrm{S} 2 \mathrm{~A}=0.750083 \backslash \mathrm{RMSD}=2.154 \mathrm{e}-09 \backslash \mathrm{RMSF}=5$. $290 \mathrm{e}-09 \backslash \mathrm{Dipole}=0 ., 0 ., 0 . \backslash \mathrm{PG}=\mathrm{D} 03 \mathrm{H} \quad[\mathrm{O}(\mathrm{C} 1), 3 \mathrm{C} 2(\mathrm{H} 1)] \backslash \backslash \mathrm{C}$

\section{$\operatorname{UCCSD}(\mathrm{T}) / 6-311 \mathrm{G}(\mathrm{d}, \mathrm{p})$}

$1 \backslash 1 \backslash G I N C-S C 23 \backslash F O p t \backslash U C C S D(T)-F C \backslash 6-311 G(d, p) \backslash C 1 H 3(2) \backslash R G B 501 \backslash 27-A u g-2002 \backslash$ $1 \backslash \backslash \# \mathrm{~N}$ UCCSD $(\mathrm{T}) \quad 6-311 \mathrm{G} *$ $* \mathrm{SCF}=\mathrm{DIRECT}$ OPT $=(\mathrm{Z}-\mathrm{MATRIX}, \mathrm{TIGHT})$ FREQ MAXDISK=1 $3107200 \backslash \backslash$ methyl $\backslash \backslash 0,2 \backslash \mathrm{C} \backslash \mathrm{X}, 1,2 . \backslash \mathrm{H}, 1, \mathrm{ch}, 2,90 . \backslash \mathrm{H}, 1, \mathrm{ch}, 2,90 ., 3,120 ., 0 \backslash \mathrm{H}, 1, \mathrm{C}$ $\mathrm{h}, 2,90 ., 3,-120 ., 0 \backslash \backslash \mathrm{ch}=1.08399744 \backslash \backslash$ Version=DEC-AXP-OSF / 1-G98RevA.11.3 S S tate $=2-\mathrm{A} 2 " \backslash \mathrm{HF}=-39.5726813 \backslash \mathrm{MP} 2=-39.7071905 \backslash \mathrm{MP} 3=-39.725534 \backslash \mathrm{MP} 4 \mathrm{D}=-39.7298$ $22 \backslash \mathrm{MP} 4 \mathrm{DQ}=-39.7276578 \backslash \mathrm{PUHF}=-39.5757157 \backslash \mathrm{PMP} 2-0=-39.7091507 \backslash \mathrm{PMP} 3-0=-39.72$ $66987 \backslash \mathrm{MP} 4 \mathrm{SDQ}=-39.7282352 \backslash \mathrm{CCSD}=-39.7290524 \backslash \mathrm{CCSD}(\mathrm{T})=-39.732253 \backslash \mathrm{S} 2=0.7617$ $42 \backslash \mathrm{S} 2-1=0.753396 \backslash \mathrm{S} 2 \mathrm{~A}=0.750077 \backslash \mathrm{RMSD}=8.070 \mathrm{e}-10 \backslash \mathrm{RMSF}=2.382 \mathrm{e}-07 \backslash \mathrm{PG}=\mathrm{D} 03 \mathrm{H} \quad[\mathrm{O}$ (C1), 3C2 (H1) ] \\@

\section{$\mathrm{CH}_{2}=\mathrm{CH}_{2}$}

\section{RHF / 6-31G (d)}

$1 \backslash 1 \backslash G I N C-S C 106 \backslash F O p t \backslash R H F \backslash 6-31 G(d) \backslash C 2 H 4 \backslash R G B 501 \backslash 23-A u g-2002 \backslash 0 \backslash \backslash \#$ HF / 6-31G * $\mathrm{SCF}=\mathrm{TIGHT}$ OPT=(TIGHT) FREQ MAXDISK=13107200\\CH2CH2 HF/6-31G(d) Opt\& Freq $\backslash \backslash 0,1 \backslash \mathrm{C}, 0 ., 0 \ldots,-0.6584681121 \backslash \mathrm{C}, 0 ., 0 ., 0.6584681121 \backslash \mathrm{H}, 0.9143401309,0$. $,-1.2256852464 \backslash \mathrm{H},-0.9143401309,0 .,-1.2256852464 \backslash \mathrm{H}, 0.9143401309,0 ., 1.22$ $56852464 \backslash \mathrm{H},-0.9143401309,0 ., 1.2256852464 \backslash \backslash$ Version=DEC-AXP $-O S F / 1-G 98 \mathrm{ReV}$ A.11.3 State $=1-A G \backslash H F=-78.0317182 \backslash R M S D=3.433 e-09 \backslash R M S F=1.178 e-06 \backslash D i p o l e=$ $0 ., 0 ., 0 . \backslash \mathrm{PG}=\mathrm{D} 02 \mathrm{H} \quad[\mathrm{C} 2 "(\mathrm{C} 1 . \mathrm{C} 1), \mathrm{SG}(\mathrm{H} 4)] \backslash \backslash \mathrm{Q}$

\section{RB3LYP/6-31G (d)}

$1 \backslash 1 \backslash G I N C-S C 74 \backslash F O p t \backslash R B 3 L Y P \backslash 6-31 G(d) \backslash C 2 H 4 \backslash R G B 501 \backslash 23-A u g-2002 \backslash 0 \backslash \backslash \#$ B3LYP / 6-31G(D) SCF=TIGHT OPT=TIGHT INT=ULTRAFINE FREQ MAXDISK=13107200 \\CH2C

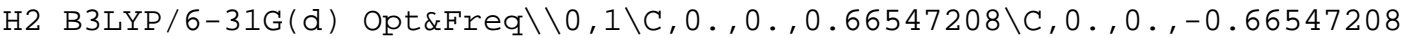
$\backslash \mathrm{H}, 0 ., 0.9235919102,1.2395837957 \backslash \mathrm{H}, 0 .,-0.9235919102,1.2395837957 \backslash \mathrm{H}, 0 ., 0$ $.9235919102,-1.2395837957 \backslash \mathrm{H}, 0 .,-0.9235919102,-1.2395837957 \backslash \backslash$ Version=DE C-AXP-OSF /1-G98RevA.11.3 State =1-AG \HF=-78.5874587\RMSD=3.405e-09 RMSF $=4.816 \mathrm{e}-06 \backslash \mathrm{Dipole}=0 ., 0 ., 0 . \backslash \mathrm{PG}=\mathrm{D} 02 \mathrm{H} \quad[\mathrm{C} 2 "(\mathrm{C} 1 . \mathrm{C} 1), \mathrm{SG}(\mathrm{H} 4)] \backslash \backslash \mathrm{C}$

\section{RB3LYP / 6-31G (2df, p)}

$1 \backslash 1 \backslash G I N C-S C 90 \backslash F O p t \backslash R B 3 L Y P \backslash 6-31 G(2 \mathrm{df}, \mathrm{p}) \backslash \mathrm{C} 2 \mathrm{H} 4 \backslash R G B 501 \backslash 29-M a y-2002 \backslash 1 \backslash \backslash \#$ B3 LYP /6-31G (2DF, P) OPT=Z-MATRIX FREQ MAXDISK=117964800 \CH2CH2 B3LYP/6-3 $1 \mathrm{G}(2 \mathrm{df}, \mathrm{p}) \quad$ Opt \&Freq $\backslash \backslash 0,1 \backslash \mathrm{C} \backslash \mathrm{C}, 1, \mathrm{r} 1 \backslash \mathrm{H}, 1, \mathrm{r} 2,2, \operatorname{ang} 1 \backslash \mathrm{H}, 1, \mathrm{r} 2,2, \operatorname{ang} 1,3,180 ., 0 \backslash$ $\mathrm{H}, 2, \mathrm{r} 2,1, \mathrm{ang} 1,3,0 ., 0 \backslash \mathrm{H}, 2, \mathrm{r} 2,1, \mathrm{ang} 1,3,180 ., 0 \backslash \backslash \mathrm{r} 1=1.32724971 \backslash \mathrm{r} 2=1.085752$ $71 \backslash$ ang $1=121.93540215 \backslash \backslash$ Version=DEC-AXP-OSF $/ 1-G 98 R e v A .11 .3 \backslash$ State $=1-A G \backslash H F$ $=-78.5954116 \backslash \mathrm{RMSD}=3.305 e-09 \backslash \mathrm{RMSF}=4.818 \mathrm{e}-05 \backslash \mathrm{Dipole}=0,0 ., 0 . \backslash \mathrm{PG}=\mathrm{D} 02 \mathrm{H} \quad[\mathrm{C} 2$ " (C1.C1), SG (H4) ] \\@

\section{RB3LYP /CC-PVTZ}

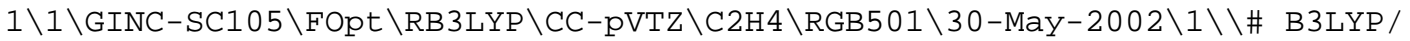
CC-PVTZ OPT=Z-MATRIX FREQ MAXDISK=2621440000\\CH2CH2 B3LYP/CC-pVTZ Opt \& Freq $\backslash \backslash 0,1 \backslash \mathrm{C} \backslash \mathrm{C}, 1, \mathrm{r} 1 \backslash \mathrm{H}, 1, \mathrm{r} 2,2$, ang $1 \backslash \mathrm{H}, 1, \mathrm{r} 2,2$, ang $1,3,180 ., 0 \backslash \mathrm{H}, 2, \mathrm{r} 2,1, \operatorname{ang} 1$ $, 3,0 ., 0 \backslash \mathrm{H}, 2, \mathrm{r} 2,1, \operatorname{ang} 1,3,180 ., 0 \backslash \backslash \mathrm{r} 1=1.32407399 \backslash \mathrm{r} 2=1.08260238 \backslash \operatorname{ang} 1=121.7$ 
$4881066 \backslash$ Version=DEC-AXP-OSF $/ 1-G 98$ RevA.11.3 State $=1-\mathrm{AG} \backslash \mathrm{HF}=-78.6231806 \backslash$ $\mathrm{RMSD}=4.318 \mathrm{e}-09 \backslash \mathrm{RMSF}=2.085 \mathrm{e}-05 \backslash \mathrm{Dipole}=0 ., 0 ., 0 . \backslash \mathrm{PG}=\mathrm{D} 02 \mathrm{H} \quad[\mathrm{C} 2 "(\mathrm{C} 1 . \mathrm{C} 1), \mathrm{SG}(\mathrm{H}$ 4) $] \backslash \backslash Q$

\section{RB3LYP /6-311+G (3df, 2p)}

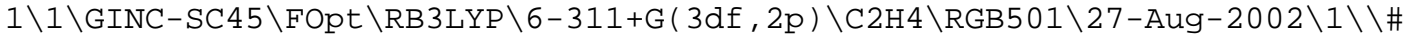
$\mathrm{B} 3 \mathrm{LYP} / 6-311+\mathrm{G}(3 \mathrm{DF}, 2 \mathrm{P}) \quad \mathrm{SCF}=\mathrm{TIGHT}$ OPT $=(\mathrm{Z}-\mathrm{MATRIX}, \mathrm{TIGHT}) \quad \mathrm{FREQ}$ MAXDISK=131 $07200 \backslash \backslash \mathrm{CH} 2 \mathrm{CH} 2$ B $3 L Y P / 6-311+\mathrm{G}(3 \mathrm{df}, 2 \mathrm{p})$ Opt \&Freq $\backslash \backslash 0,1 \backslash \mathrm{C} \backslash \mathrm{C}, 1, \mathrm{r} 1 \backslash \mathrm{H}, 1, \mathrm{r} 2,2$, an $\mathrm{g} 1 \backslash \mathrm{H}, 1, \mathrm{r} 2,2, \mathrm{ang} 1,3,180 ., 0 \backslash \mathrm{H}, 2, \mathrm{r} 2,1, \operatorname{ang} 1,3,0 ., 0 \backslash \mathrm{H}, 2, \mathrm{r} 2,1, \operatorname{ang} 1,3,180 ., 0 \backslash$ $\backslash r 1=1.32476023 \backslash r 2=1.08256577 \backslash$ ang $1=121$. $77444493 \backslash \backslash$ Version=DEC-AXP $-O S F / 1-$ G98RevA.11.3 \State $=1-\mathrm{AG} \backslash \mathrm{HF}=-78.6211961 \backslash \mathrm{RMSD}=7.412 \mathrm{e}-09 \backslash \mathrm{RMSF}=3.121 \mathrm{e}-08 \backslash \mathrm{D}$ ipole $=0 ., 0 ., 0 . \backslash \mathrm{PG}=\mathrm{D} 02 \mathrm{H} \quad[\mathrm{C} 2 "(\mathrm{C} 1 . \mathrm{C} 1), \mathrm{SG}(\mathrm{H} 4)] \backslash \backslash \mathrm{C}$

\section{RMPW1K/6-31+G (d, P)}

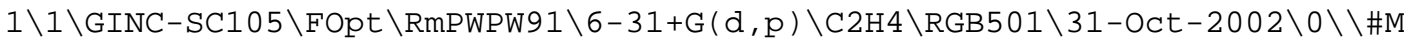
PWPW91 6-31+G** IOP $(5 / 45=10000428) \quad \operatorname{IOP}(5 / 46=05720572) \quad$ IOP $(5 / 47=1000100$

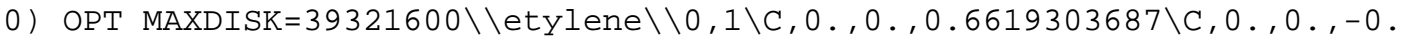
$6619303687 \backslash \mathrm{H}, 0 ., 0.919708013,1.228941991 \backslash \mathrm{H}, 0 .,-0.919708013,1.228941991 \backslash$ $\mathrm{H}, 0 ., 0.919708013,-1.228941991 \backslash \mathrm{H}, 0 .,-0.919708013,-1.228941991 \backslash \backslash$ Version= DEC-AXP-OSF /1-G98RevA.11.3\State $=1-A G \backslash H F=-78.5700179 \backslash R M S D=1.567 e-09 \backslash R M$ $\mathrm{SF}=4.139 \mathrm{e}-05 \backslash \mathrm{Dipole}=0 ., 0 ., 0 . \backslash \mathrm{PG}=\mathrm{D} 02 \mathrm{H} \quad[\mathrm{C} 2$ " (C1.C1), SG (H4) $] \backslash \backslash \mathrm{Q}$

\section{RMP2/6-31G (d)}

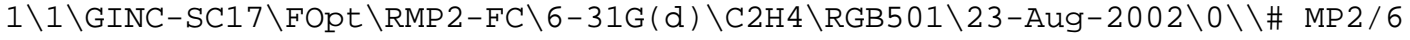

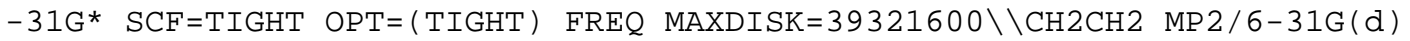
Opt\&Freq $\backslash \backslash 0,1 \backslash \mathrm{C}, 0.0 \ldots,-0.668185704 \backslash \mathrm{C}, 0.0 ., 0.668185704 \backslash \mathrm{H}, 0.9232704051$ $, 0 .,-1.2382924048 \backslash \mathrm{H},-0.9232704051,0 .,-1.2382924048 \backslash \mathrm{H}, 0.9232704051,0 ., 1$ $.2382924048 \backslash \mathrm{H},-0.9232704051,0 ., 1.2382924048 \backslash \backslash$ Version=DEC-AXP-OSF / 1-G98 RevA.11.3 State $=1-\mathrm{AG} \backslash \mathrm{HF}=-78.0309816 \backslash \mathrm{MP} 2=-78.2850278 \backslash \mathrm{RMSD}=1.885 e-09 \backslash \mathrm{RMS}$ $\mathrm{F}=2.475 e-06 \backslash \mathrm{Dipole}=0 ., 0 ., 0 . \backslash \mathrm{PG}=\mathrm{D} 02 \mathrm{H} \quad[\mathrm{C} 2$ " (C1.C1), SG (H4) ] \\@

\section{RQCISD/6-31G (d)}

$1 \backslash 1 \backslash G I N C-S C 100 \backslash F O p t \backslash R Q C I S D-F C \backslash 6-31 G(d) \backslash C 2 H 4 \backslash R G B 501 \backslash 19-S e p-2002 \backslash 1 \backslash \backslash \# N Q$ CISD 6-31G* FOPT=(Z-MATRIX, TIGHT) FREQ MAXDISK=39321600 \CH2CH2 QCISD/ 6-31g* Opt\&Freq $\backslash \backslash 0,1 \backslash \mathrm{C} \backslash \mathrm{C}, 1, \mathrm{r} 1 \backslash \mathrm{H}, 1, \mathrm{r} 2,2$, ang $1 \backslash \mathrm{H}, 1, \mathrm{r} 2,2$, ang $1,3,180 ., 0 \backslash \mathrm{H}, 2$ $, r 2,1, \operatorname{ang} 1,3,0 ., 0 \backslash H, 2, r 2,1, \operatorname{ang} 1,3,180 ., 0 \backslash \backslash r 1=1.33826095 \backslash r 2=1.08834861 \backslash$ ang $1=121.8191162 \backslash \backslash$ Version=DEC-AXP $-\mathrm{OSF} / 1-\mathrm{G} 98 \mathrm{RevA} .11 .3 \backslash$ State $=1-\mathrm{AG} \backslash \mathrm{HF}=-78$ $.0306753 \backslash \mathrm{MP} 2=-78.2849916 \backslash \mathrm{MP} 3=-78.3059336 \backslash \mathrm{MP} 4 \mathrm{D}=-78.3134712 \backslash \mathrm{MP} 4 \mathrm{DQ}=-78.30$ $86925 \backslash \mathrm{MP} 4 \mathrm{SDQ}=-78.3114119 \backslash \mathrm{QCISD}=-78.3133516 \backslash \mathrm{RMSD}=3.235 \mathrm{e}-09 \backslash \mathrm{RMSF}=2.826 \mathrm{e}-$ $06 \backslash \mathrm{Dipole}=0 ., 0 ., 0 . \backslash \mathrm{PG}=\mathrm{D} 02 \mathrm{H} \quad[\mathrm{C} 2 "(\mathrm{C} 1 . \mathrm{C} 1), \mathrm{SG}(\mathrm{H} 4)] \backslash \backslash \mathrm{C}$

\section{$\operatorname{RCCSD}(\mathrm{T}) / 6-311 \mathrm{G}(\mathrm{d}, \mathrm{p})$}

$1 \backslash 1 \backslash G I N C-S C 81 \backslash F O p t \backslash U C C S D(T)-F C \backslash 6-311 G(d, p) \backslash C 2 H 4 \backslash R G B 501 \backslash 23-S e p-2002 \backslash 1 \backslash \backslash$

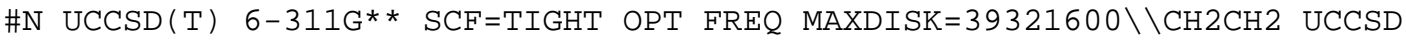

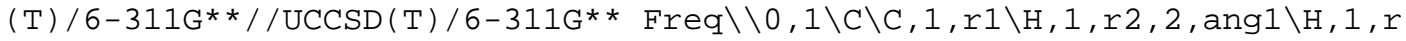
2, 2, ang1, 3, $180 ., 0 \backslash \mathrm{H}, 2, \mathrm{r2}, 1, \mathrm{ang} 1,3,0 ., 0 \backslash \mathrm{H}, 2, \mathrm{r2}, 1, \operatorname{ang} 1,3,180 ., 0 \backslash \backslash \mathrm{r} 1=1.34$ $360932 \backslash r 2=1.08861271 \backslash$ ang $1=121.53854556 \backslash \backslash$ Version=DEC-AXP-OSF $/ 1-G 98$ RevA. $11.3 \backslash$ State $=1-\mathrm{AG} \backslash \mathrm{HF}=-78.0533978 \backslash \mathrm{MP} 2=-78.3442088 \backslash \mathrm{MP} 3=-78.3673235 \backslash \mathrm{MP} 4 \mathrm{D}=-7$ $8.3755251 \backslash \mathrm{MP} 4 \mathrm{DQ}=-78.3689975 \backslash \mathrm{PUHF}=-78.0533978 \backslash \mathrm{PMP} 2-0=-78.3442088 \backslash \mathrm{PMP} 3-0$ $=-78.3673235 \backslash \mathrm{MP} 4 \mathrm{SDQ}=-78.3715875 \backslash \mathrm{CCSD}=-78.3723454 \backslash \mathrm{CCSD}(\mathrm{T})=-78.384053 \backslash \mathrm{S} 2$ $=0 . \backslash \mathrm{S} 2-1=0 . \backslash \mathrm{S} 2 \mathrm{~A}=0 . \backslash \mathrm{RMSD}=9.206 \mathrm{e}-09 \backslash \mathrm{RMSF}=1.591 \mathrm{e}-04 \backslash \mathrm{PG}=\mathrm{D} 02 \mathrm{H} \quad[\mathrm{C} 2 "(\mathrm{C} 1 . \mathrm{C} 1), \mathrm{S}$ $\mathrm{G}(\mathrm{H} 4)] \backslash \backslash \mathrm{e}$

\section{$\cdot \mathrm{CH}_{3}---\mathrm{CH}_{2}=\mathrm{CH}_{2}$}

\section{$\mathrm{UHF} / 6-31 \mathrm{G}(\mathrm{d})$}

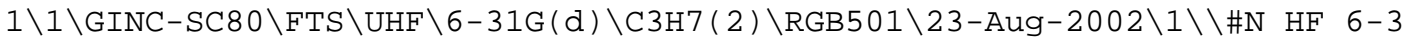
$1 G$ * SCF $=$ TIGHT OPT $=(T S$, NOEIGENTEST, Z-MATRIX, TIGHT) TEST FREQ MAXDISK=13

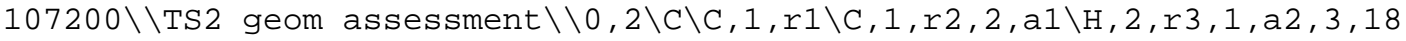
$0 ., 0 \backslash \mathrm{H}, 2, \mathrm{r} 4,1, \mathrm{a} 3,4, \mathrm{~d} 1,0 \backslash \mathrm{H}, 2, \mathrm{r} 4,1, \mathrm{a} 3,4,-\mathrm{d} 1,0 \backslash \mathrm{H}, 1, \mathrm{r} 5,3, \mathrm{a} 4,2, \mathrm{~d} 2,0 \backslash \mathrm{H}, 1, \mathrm{r} 5$, 
3, a4 , 2, $-\mathrm{d} 2,0 \backslash \mathrm{H}, 3, \mathrm{r} 6,1, \mathrm{a} 5,2, \mathrm{~d} 3,0 \backslash \mathrm{H}, 3, \mathrm{r} 6,1, \mathrm{a} 5,2,-\mathrm{d} 3,0 \backslash \mathrm{r} 1=2.24565522 \backslash \mathrm{r} 2=$ $1.38162693 \backslash r 3=1.07721228 \backslash r 4=1.0759145 \backslash r 5=1.07505938 \backslash r 6=1.07533545 \backslash a 1=1$ $09.0767733 \backslash a 2=100.9940995 \backslash a 3=102.71031022 \backslash a 4=119.70743478 \backslash a 5=121.34233$ $841 \backslash \mathrm{d} 1=119.83475021 \backslash \mathrm{d} 2=103.20905249 \backslash \mathrm{d} 3=86.5453928 \backslash \backslash$ Version=DEC $-\mathrm{AXP}-\mathrm{OSF}$ /1-G98RevA.11.3 State $=2-A^{\prime} \backslash \mathrm{HF}=-117.5756924 \backslash \mathrm{S} 2=1.028565 \backslash \mathrm{S} 2-1=0 . \backslash \mathrm{S} 2 \mathrm{~A}=0.7$ $6081 \backslash \mathrm{RMSD}=6.773 e-09 \backslash \mathrm{RMSF}=1.394 \mathrm{e}-06 \backslash \mathrm{Dipole}=-0.0494069,0,0.0612636 \backslash \mathrm{PG}=\mathrm{C}$ $\mathrm{S} \quad[\mathrm{SG}(\mathrm{C} 3 \mathrm{H} 1), \mathrm{X}(\mathrm{H} 6)] \backslash \backslash @$

\section{UB3LYP/6-31G (d)}

$1 \backslash 1 \backslash G I N C-S C 17 \backslash F T S \backslash U B 3 L Y P \backslash 6-31 G(d) \backslash C 3 H 7(2) \backslash R G B 501 \backslash 23-A u g-2002 \backslash 0 \backslash \backslash \#$ B3LY $\mathrm{P} / 6-31 \mathrm{G} * \mathrm{SCF}=\mathrm{TIGHT}$ OPT $=(\mathrm{TS}, \mathrm{NOEIGENTEST}, \mathrm{CALCFC}, \mathrm{TIGHT})$ FREQ MAXDISK=1310 $7200 \backslash \backslash \mathrm{CH} 2 \mathrm{CH} 2 \mathrm{~B} 3 \mathrm{LYP} / 6-31 \mathrm{G}(\mathrm{d})$ Opt\&Freq $\backslash \backslash 0,2 \backslash \mathrm{C},-0.8121054645,0 .,-0.131646$ $4239 \backslash \mathrm{C},-0.8053024498,0 ., 1.223851904 \backslash \mathrm{C}, 1.4055309203,0 \ldots,-0.951853018 \backslash \mathrm{H}, 1$ $.1967512348,0 .,-2.0176023429 \backslash \mathrm{H},-0.7682356122,-0.9243017713,1.794348012$ $3 \backslash \mathrm{H},-0.7682356122,0.9243017713,1.7943480123 \backslash \mathrm{H},-0.9660003017,0.92011418$ $21,-0.6878676819 \backslash \mathrm{H},-0.9660003017,-0.9201141821,-0.6878676819 \backslash \mathrm{H}, 1.77149$ $12786,-0.9244100053,-0.5187365451 \backslash \mathrm{H}, 1.7714912786,0.9244100053,-0.51873$ $65451 \backslash \backslash$ Version=DEC-AXP-OSF /1-G98RevA.11.3 State=2-A ' \HF=-118.4187742 \S $2=0.780138 \backslash S 2-1=0 . \backslash S 2 A=0.750184 \backslash R M S D=6.716 e-09 \backslash R M S F=6.072 e-06 \backslash D i p o l e=0$ $.0296824,0 .,-0.0698267 \backslash \mathrm{PG}=\mathrm{CS} \quad[\mathrm{SG}(\mathrm{C} 3 \mathrm{H} 1), \mathrm{X}(\mathrm{H} 6)] \backslash \backslash \mathrm{C}$

\section{UB3LYP / 6-31G (2df, p)}

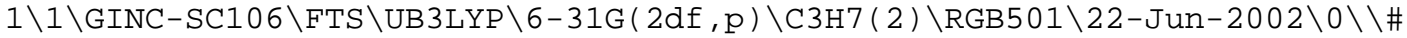
UB3LYP / 6-31G $(2 \mathrm{DF}, \mathrm{P}) \quad \mathrm{OPT}=(\mathrm{TS}, \mathrm{NOEIGENTEST}, \mathrm{CALCFC}) \quad \mathrm{FREQ}$ MAXDISK=39321600 $\backslash \backslash \mathrm{CH} 2 \mathrm{CH} 2 \mathrm{UB} 3 \mathrm{LYP} / 6-31 \mathrm{G}(2 \mathrm{df}, \mathrm{p})$ Opt\&Freq $\backslash \backslash 0,2 \backslash \mathrm{C},-0.8008777794,0,-0.15415$ $60041 \backslash \mathrm{C},-0.831072971,0 ., 1.1988611253 \backslash \mathrm{C}, 1.418365538,0 .,-0.9103307804 \backslash \mathrm{H}$, $1.233914785,0 .,-1.9790182432 \backslash \mathrm{H},-0.8107886591,-0.9228019984,1.769642718$ $9 \backslash \mathrm{H},-0.8107886591,0.9228019984,1.7696427189 \backslash \mathrm{H},-0.9406919665,0.91830565$ $4,-0.7141494866 \backslash \mathrm{H},-0.9406919665,-0.918305654,-0.7141494866 \backslash \mathrm{H}, 1.7752788$ $702,-0.9224890031,-0.4691071333 \backslash \mathrm{H}, 1.7752788702,0.9224890031,-0.4691071$ 333 \\Version=DEC-AXP-OSF /1-G98RevA.11.3 State $=2-A^{\prime} \backslash H F=-118.4317726 \backslash S 2=$ $0.780522 \backslash S 2-1=0 . \backslash S 2 A=0.750192 \backslash R M S D=7.011 e-09 \backslash R M S F=8.887 e-05 \backslash D i p o l e=0.0$ $275072,0 .,-0.0688979 \backslash \mathrm{PG}=\mathrm{CS} \quad[\mathrm{SG}(\mathrm{C} 3 \mathrm{H} 1), \mathrm{X}(\mathrm{H} 6)] \backslash \backslash \mathrm{C}$

\section{UB3LYP / CC-PVTZ}

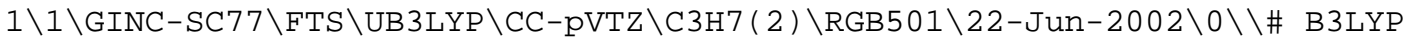
/CC-PVTZ OPT=(TS, NOEIGENTEST, CALCFC) FREQ MAXDISK=262144000\\CH2CH2 B3

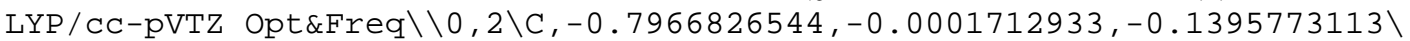
$\mathrm{C},-0.7924958251,0.0000311945,1.2125333415 \backslash \mathrm{C}, 1.3831087809,0.0001190936$, $-0.935227513 \backslash \mathrm{H}, 1.1746017559,0.0004250568,-1.9960339811 \backslash \mathrm{H},-0.7574886895$ $,-0.9211362058,1.778761158 \backslash \mathrm{H},-0.7577975316,0.9213780323,1.7784881546 \backslash \mathrm{H}$ $,-0.9593456523,0.9161455526,-0.6895817892 \backslash \mathrm{H},-0.9590357538,-0.916714326$ $9,-0.6893019943 \backslash \mathrm{H}, 1.74781384,-0.9202643189,-0.5046319617 \backslash \mathrm{H}, 1.747670223$ $2,0.9202922407,-0.5040706897 \backslash \backslash$ Version=DEC-AXP-OSF / 1-G98RevA.11.3\HF=-1 $18.4723095 \backslash \mathrm{S} 2=0.778674 \backslash \mathrm{S} 2-1=0 . \backslash \mathrm{S} 2 \mathrm{~A}=0.750169 \backslash \mathrm{RMSD}=5.504 \mathrm{e}-09 \backslash \mathrm{RMSF}=5.626 \mathrm{e}$ $-05 \backslash \mathrm{Dipole}=0.0129783,-0.000004,-0.0614079 \backslash \mathrm{PG}=\mathrm{C} 01 \quad[\mathrm{X}(\mathrm{C} 3 \mathrm{H} 7)] \backslash \backslash @$

\section{UB3LYP / 6-311+G (3df, 2p)}

$1 \backslash 1 \backslash G I N C-S C 63 \backslash F T S \backslash U B 3 L Y P \backslash 6-311+G(3 d f, 2 p) \backslash C 3 H 7(2) \backslash R G B 501 \backslash 24-A u g-2002 \backslash 0 \backslash$ $\backslash \#$ UB3LYP $6-311+G(3 \mathrm{DF}, 2 \mathrm{P}) \quad \mathrm{SCF}=\mathrm{TIGHT}$ OPT=(TS, NOEIGENTEST, CALCFC, TIGHT)

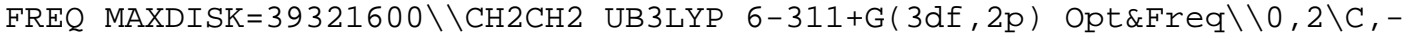
$0.7973790215,0 \ldots,-0.1461863455 \backslash \mathrm{C},-0.7975868437,0 ., 1.2064428896 \backslash \mathrm{C}, 1.3886$ $920788,0 .,-0.9244790284 \backslash \mathrm{H}, 1.186050977,0 \ldots,-1.9862910972 \backslash \mathrm{H},-0.7651331863$ $,-0.9209272087,1.7731731761 \backslash \mathrm{H},-0.7651331863,0.9209272087,1.7731731761 \backslash$ $\mathrm{H},-0.9567116553,0.9162355173,-0.6973878515 \backslash \mathrm{H},-0.9567116553,-0.91623551$ $73,-0.6973878515 \backslash \mathrm{H}, 1.7476407123,-0.9206439823,-0.4899723232 \backslash \mathrm{H}, 1.747640$ $7123,0.9206439823,-0.4899723232 \backslash \backslash$ Version=DEC-AXP-OSF / 1-G98RevA.11.3 St ate $=2-A^{\prime} \backslash \mathrm{HF}=-118.4691472 \backslash \mathrm{S} 2=0.778198 \backslash \mathrm{S} 2-1=0 . \backslash \mathrm{S} 2 \mathrm{~A}=0.750165 \backslash \mathrm{RMSD}=7.267 \mathrm{e}-$ $09 \backslash \mathrm{RMSF}=2.311 \mathrm{e}-06 \backslash \mathrm{Dipole}=0.0088836,0 .,-0.0617824 \backslash \mathrm{PG}=\mathrm{CS} \quad[\mathrm{SG}(\mathrm{C} 3 \mathrm{H} 1), \mathrm{X}(\mathrm{H} 6)$ 
]$\backslash \backslash @$

\section{UMPW1K/6-31+G (d,p)}

$1 \backslash 1 \backslash G I N C-S C 66 \backslash F T S \backslash U m P W P W 91 \backslash 6-31+G(d, p) \backslash C 3 H 7(2) \backslash R G B 501 \backslash 01-N o v-2002 \backslash 0 \backslash \backslash \#$ MPWPW91 6-31+G** IOP (5/45=10000428) IOP $(5 / 46=05720572) \quad$ IOP $(5 / 47=100010$ $00)$ MAXDISK=39321600 OPT $=(\mathrm{TS}, \mathrm{CALCFC}, \mathrm{NOEIGENTEST}) \backslash \backslash \mathrm{TS}$ ET $\backslash \backslash 0,2 \backslash \mathrm{C},-0.0069$ $548954,0.8180094522,0 . \backslash \mathrm{C}, 1.3225317072,0.5855096443,0 . \backslash \mathrm{C},-1.147547288$,$1.2219276345,0 . \backslash \mathrm{H},-2.1563027907,-0.8381427703,0 . \backslash \mathrm{H}, 1.8725451669,0.4589$ $426326,0.9206602989 \backslash \mathrm{H}, 1.8725451669,0.4589426326,-0.9206602989 \backslash \mathrm{H},-0.525$ $6213688,1.0545795666,-0.9169434227 \backslash \mathrm{H},-0.5256213688,1.0545795666,0.9169$ $434227 \backslash \mathrm{H},-0.7728609738,-1.6392252,0.9203982007 \backslash \mathrm{H},-0.7728609738,-1.6392$ $252,-0.9203982007 \backslash \backslash$ Version=DEC-AXP-OSF / 1-G98RevA. 11.3 State $=2-A^{\prime} \backslash \mathrm{HF}=-1$ $18.3958645 \backslash \mathrm{S} 2=0.812651 \backslash \mathrm{S} 2-1=0 . \backslash \mathrm{S} 2 \mathrm{~A}=0.750602 \backslash \mathrm{RMSD}=5.247 \mathrm{e}-09 \backslash \mathrm{RMSF}=1.020 \mathrm{e}$ $-05 \backslash$ Dipole $=-0.0786297,-0.0269125,0 . \backslash \mathrm{PG}=\mathrm{CS} \quad[\mathrm{SG}(\mathrm{C} 3 \mathrm{H} 1), \mathrm{X}(\mathrm{H} 6)] \backslash \backslash \mathrm{e}$

\section{UMP 2/6-31G (d)}

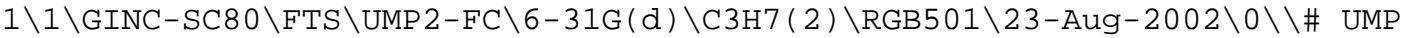
$2 / 6-31 \mathrm{G} * \mathrm{SCF}=\mathrm{TIGHT}$ OPT $=(\mathrm{TS}, \mathrm{NOEIGENTEST}, \mathrm{CALCFC}, \mathrm{TIGHT}) \quad$ FREQ MAXDISK=3932 $1600 \backslash \backslash \mathrm{CH} 2 \mathrm{CH} 2 \mathrm{UMP} 2(\mathrm{FU}) / 6-31 \mathrm{G}(\mathrm{d})$ Opt\&Freq $\backslash \backslash 0,2 \backslash \mathrm{C},-0.7814488501,0.0004239$ $461,-0.1469547485 \backslash \mathrm{C},-0.7757948928,0.0002295286,1.197282067 \backslash \mathrm{C}, 1.3463083$ $954,-0.0005650745,-0.9127069249 \backslash \mathrm{H}, 1.1695691507,-0.0003542491,-1.982411$ $4964 \backslash \mathrm{H},-0.7332378789,-0.9219873652,1.7655126723 \backslash \mathrm{H},-0.7322632281,0.9222$ $389235,1.7657754917 \backslash \mathrm{H},-0.9505049778,0.9183794921,-0.69830735 \backslash \mathrm{H},-0.9514$ $702055,-0.9171959111,-0.6985684332 \backslash \mathrm{H}, 1.7313018977,-0.9191577197,-0.488$ $9666535 \backslash \mathrm{H}, 1.732217327,0.9175464279,-0.4887565921 \backslash \backslash$ Version=DEC-AXP $-O S F /$ $1-G 98 R e v A .11 .3 \backslash \mathrm{HF}=-117.5743974 \backslash \mathrm{MP} 2=-117.9312439 \backslash \mathrm{PUHF}=-117.592189 \backslash \mathrm{PMP} 2-$ $0=-117.9465795 \backslash S 2=0.990354 \backslash S 2-1=0.922549 \backslash S 2 A=0.758812 \backslash R M S D=4.255 e-09 \backslash R$ $\mathrm{MSF}=6.834 \mathrm{e}-07 \backslash \mathrm{Dipole}=0.0478569,-0.0000098,-0.0971329 \backslash \mathrm{PG}=\mathrm{C} 01 \quad[\mathrm{X}(\mathrm{C} 3 \mathrm{H} 7)] \backslash$ $\backslash$ Q

\section{UQCISD/6-31G (d)}

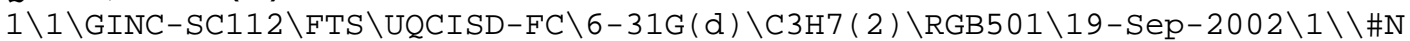
QCISD 6-31G* FOPT=(TS,Z-MATRIX, TIGHT, NOEIGENTEST) FREQ TEST MAXDISK=6

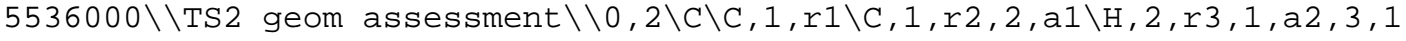
$80 ., 0 \backslash \mathrm{H}, 2, \mathrm{r} 4,1, \mathrm{a} 3,4, \mathrm{~d} 1,0 \backslash \mathrm{H}, 2, \mathrm{r} 4,1, \mathrm{a} 3,4,-\mathrm{d} 1,0 \backslash \mathrm{H}, 1, \mathrm{r} 5,3, \mathrm{a} 4,2, \mathrm{~d} 2,0 \backslash \mathrm{H}, 1, \mathrm{r} 5$ $, 3, \mathrm{a} 4,2,-\mathrm{d} 2,0 \backslash \mathrm{H}, 3, \mathrm{r} 6,1, \mathrm{a} 5,2, \mathrm{~d} 3,0 \backslash \mathrm{H}, 3, \mathrm{r} 6,1, \mathrm{a} 5,2,-\mathrm{d} 3,0 \backslash \backslash \mathrm{r} 1=2.27231968 \backslash \mathrm{r} 2$ $=1.36700426 \backslash r 3=1.08819496 \backslash r 4=1.08673589 \backslash r 5=1.08765982 \backslash r 6=1.08782636 \backslash a 1$ $=109.53122811 \backslash a 2=100.39525709 \backslash a 3=101.45604463 \backslash a 4=120.27663792 \backslash a 5=121.5$ $39714 \backslash \mathrm{d} 1=119.97480827 \backslash \mathrm{d} 2=101.50405094 \backslash \mathrm{d} 3=86.90924411 \backslash \backslash$ Version=DEC $-\mathrm{AXP}-$

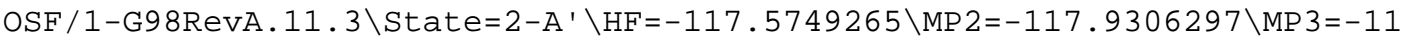
$7.968586 \backslash M P 4 D=-117.9792401 \backslash M P 4 D Q=-117.9737865 \backslash P U H F=-117.594011 \backslash P M P 2-0=$ $-117.9472093 \backslash \mathrm{PMP} 3-0=-117.981531 \backslash \mathrm{MP} 4 \mathrm{SDQ}=-117.9786867 \backslash \mathrm{QCISD}=-117.9884099$ $\backslash S 2=1.018928 \backslash S 2-1=0.946664 \backslash S 2 A=0.760701 \backslash R M S D=8.322 e-09 \backslash R M S F=6.976 e-07 \backslash$ Dipole $=-0.0552007,0.0 .0675931 \backslash P G=C S \quad[S G(C 3 H 1), X(H 6)] \backslash \backslash @$

\section{$\operatorname{UCCSD}(T) / 6-311 G(d, p)$}

$1 \backslash 1 \backslash G I N C-S C 84 \backslash F T S \backslash U C C S D(T)-F C \backslash 6-311 G(d, p) \backslash C 3 H 7(2) \backslash R G B 501 \backslash 11-0 c t-2002 \backslash 1$ $\backslash \backslash \# \operatorname{CCSD}(\mathrm{T}) / 6-311 \mathrm{G} * \star \mathrm{SCF}=\mathrm{DIRECT}$ OPT=(TS,EF, Z-MATRIX) FREQ MAXDISK=2621 $44000 \backslash \backslash$ reoptimizacion y Freqs CCSD (T)/6-311G** on Aces geom $\backslash \backslash 0,2 \backslash C \backslash C, 1$ , $\mathrm{B} 1 \backslash \mathrm{C}, 1, \mathrm{~B} 2,2, \mathrm{~A} 1 \backslash \mathrm{H}, 2, \mathrm{~B} 3,1, \mathrm{~A} 2,3,180 ., 0 \backslash \mathrm{H}, 2, \mathrm{~B} 4,1, \mathrm{~A} 3,4, \mathrm{D} 1,0 \backslash \mathrm{H}, 2, \mathrm{~B} 4,1, \mathrm{~A} 3,4$, $-\mathrm{D} 1,0 \backslash \mathrm{H}, 1, \mathrm{~B} 5,2, \mathrm{~A} 4,3, \mathrm{D} 2,0 \backslash \mathrm{H}, 1, \mathrm{~B} 5,2, \mathrm{~A} 4,3,-\mathrm{D} 2,0 \backslash \mathrm{H}, 3, \mathrm{~B} 6,1, \mathrm{~A} 5,2, \mathrm{D} 3,0 \backslash \mathrm{H}, 3, \mathrm{~B} 6$ $, 1, A 5,2,-D 3,0 \backslash \backslash B 1=2.28890735 \backslash B 2=1.36812294 \backslash B 3=1.0886586 \backslash B 4=1.08725254 \backslash$ $\mathrm{B} 5=1.08809775 \backslash \mathrm{B} 6=1.0881489 \backslash \mathrm{A} 1=109.29988576 \backslash \mathrm{A} 2=99.87795506 \backslash \mathrm{A} 3=100.83806$ $811 \backslash A 4=89.21542439 \backslash A 5=121.26203625 \backslash D 1=120.01232642 \backslash D 2=-121.86368323 \backslash D 3$ $=86.91048876 \backslash \backslash$ Version=DEC-AXP-OSF /1-G98RevA.11.3 State $=2-A^{\prime} \backslash H F=-117.60$ $948 \backslash \mathrm{MP} 2=-118.0294616 \backslash \mathrm{MP} 3=-118.072175 \backslash \mathrm{MP} 4 \mathrm{D}=-118.0839906 \backslash \mathrm{MP} 4 \mathrm{DQ}=-118.0758$ $742 \backslash \mathrm{PUHF}=-117.6280243 \backslash \mathrm{PMP} 2-0=-118.0455666 \backslash \mathrm{PMP} 3-0=-118.0846566 \backslash \mathrm{MP} 4 \mathrm{SDQ}=-$ $118.0807325 \backslash \mathrm{CCSD}=-118.0880105 \backslash \mathrm{CCSD}(\mathrm{T})=-118.1043729 \backslash \mathrm{S} 2=1.013676 \backslash \mathrm{S} 2-1=0$. $94267 \backslash \mathrm{S} 2 \mathrm{~A}=0.760491 \backslash \mathrm{RMSD}=6.270 \mathrm{e}-09 \backslash \mathrm{RMSF}=6.805 \mathrm{e}-05 \backslash \mathrm{PG}=\mathrm{CS} \quad[\mathrm{SG}(\mathrm{C} 3 \mathrm{H} 1), \mathrm{X}(\mathrm{H} 6)$ 
]$\backslash \backslash @$

\section{$\mathrm{CH}_{3}-\mathrm{CH}_{2}-\mathrm{CH}_{2} \cdot$}

\section{$\mathrm{UHF} / 6-31 \mathrm{G}(\mathrm{d})$}

$1 \backslash 1 \backslash G I N C-S C 112 \backslash F O p t \backslash U H F \backslash 6-31 G(d) \backslash C 3 H 7(2) \backslash R G B 501 \backslash 22-J u n-2002 \backslash 0 \backslash \backslash \#$ UHF / $-31 \mathrm{G} *$ OPT FREQ MAXDISK=13107200 \\H2CH2 UHF/6-31G(d) Opt\&Freq $\backslash \backslash 0,2 \backslash \mathrm{C},-$ $0.5001280009,0 \ldots,-0.2944798298 \backslash \mathrm{C},-0.6044216655,0 ., 1.2033928506 \backslash \mathrm{C}, 0.9507$ $664237,0 .,-0.799201746 \backslash \mathrm{H}, 0.9831868705,0 .,-1.8846706354 \backslash \mathrm{H},-0.5026555695$ $,-0.918168754,1.7550289285 \backslash \mathrm{H},-0.5026555695,0.918168754,1.7550289285 \backslash \mathrm{H}$, $-1.0130662862,0.8709818039,-0.6938947559 \backslash \mathrm{H},-1.0130662862,-0.8709818039$ $,-0.6938947559 \backslash \mathrm{H}, 1.4854781488,-0.877051006,-0.4479326794 \backslash \mathrm{H}, 1.485478148$ $8,0.877051006,-0.4479326794 \backslash \backslash$ Version=DEC-AXP-OSF /1-G98RevA.11.3\State= $2-A^{\prime} \backslash H F=-117.631433 \backslash S 2=0.762364 \backslash S 2-1=0 . \backslash S 2 A=0.750102 \backslash R M S D=4.131 e-09 \backslash R M$ $\mathrm{SF}=1.603 e-04 \backslash \mathrm{Dipole}=0.0686566,0 .,-0.0440716 \backslash \mathrm{PG}=\mathrm{CS} \quad[\mathrm{SG}(\mathrm{C} 3 \mathrm{H} 1), \mathrm{X}(\mathrm{H} 6)] \backslash \backslash @$

\section{UB3LYP / 6-31G (d)}

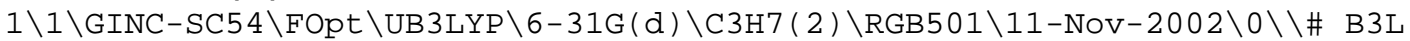

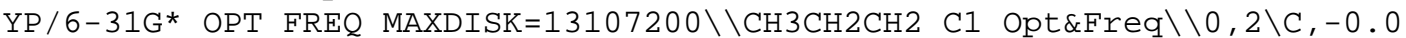
$786818346,0.5545971888,0.0535328618 \backslash \mathrm{C},-1.3053602246,-0.2910141762,-0.0$ $291135868 \backslash \mathrm{C}, 1.2272829113,-0.247670495,-0.0431132887 \backslash \mathrm{H}, 2.1028011425,0.4$ $041052573,0.0531453484 \backslash \mathrm{H},-1.3055801836,-1.3043303175,0.3632827032 \backslash \mathrm{H},-2$ $.2602674092,0.1328179499,-0.3242018731 \backslash \mathrm{H},-0.0732417371,1.1211963362,1$. $0043941973 \backslash \mathrm{H},-0.10283005,1.3233246388,-0.7322485662 \backslash \mathrm{H}, 1.2967048243,-0$. $7707100669,-1.0033584233 \backslash \mathrm{H}, 1.2829683007,-1.0018789033,0.751150696 \backslash \backslash$ Ver

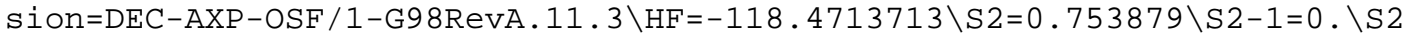
$\mathrm{A}=0.75001 \backslash \mathrm{RMSD}=5.437 \mathrm{e}-09 \backslash \mathrm{RMSF}=4.553 \mathrm{e}-05 \backslash \mathrm{Dipole}=0.0489854,0.0717907,0.0$ $473236 \backslash P G=\mathrm{C} 01 \quad[\mathrm{X}(\mathrm{C} 3 \mathrm{H} 7)] \backslash \backslash €$

\section{UB3LYP / 6-31G (2df, p)}

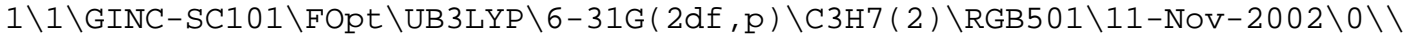
\# B3LYP/6-31G(2DF, P) OPT SCF=TIGHT FREQ MAXDISK=39321600 \CH3CH2CH2 B3

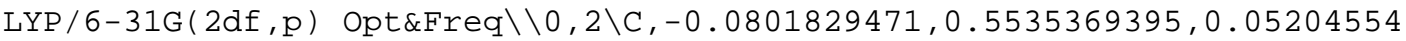
$38 \backslash \mathrm{C},-1.3037195625,-0.2919807336,-0.0334228302 \backslash \mathrm{C}, 1.2267374709,-0.24547$ $11064,-0.0370187515 \backslash \mathrm{H}, 2.0996660048,0.4074395414,0.0588883113 \backslash \mathrm{H},-1.2909$ $909362,-1.3196549953,0.3145079326 \backslash \mathrm{H},-2.2638146968,0.1380349253,-0.2951$ $513978 \backslash \mathrm{H},-0.0806439688,1.1237123451,0.9995147972 \backslash \mathrm{H},-0.1024431407,1.321$ $7529984,-0.7333296285 \backslash \mathrm{H}, 1.3002435749,-0.7722762807,-0.9936584745 \backslash \mathrm{H}, 1.2$ $809733947,-0.9955191316,0.7596046871 \backslash \backslash$ Version=DEC-AXP-OSF / 1-G98RevA. 11 $.3 \backslash \mathrm{HF}=-118.4824804 \backslash \mathrm{S} 2=0.754081 \backslash \mathrm{S} 2-1=0 . \backslash \mathrm{S} 2 \mathrm{~A}=0.75001 \backslash \mathrm{RMSD}=8.951 \mathrm{e}-09 \backslash \mathrm{RMSF}$ $=2.767 e-06 \backslash \mathrm{Dipole}=0.0569078,0.0736747,0.0424533 \backslash \mathrm{PG}=\mathrm{C} 01 \quad[\mathrm{X}(\mathrm{C} 3 \mathrm{H} 7)] \backslash \backslash \mathrm{Q}$

\section{UB3LYP /CC-pVTZ}

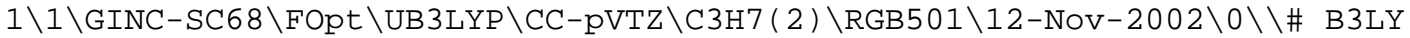
P/CC-PVTZ OPT FREQ SCF=TIGHT MAXDISK=39321600\\CH3CH2CH2 B3LYP/CC-pVTZ Opt\&Freq $\backslash \backslash 0,2 \backslash C,-0.0794241123,0.5510155957,0.0490011779 \backslash \mathrm{C},-1.30240792$ $6,-0.2887823781,-0.029298179 \backslash \mathrm{C}, 1.2246791297,-0.2458031523,-0.035250528$ $7 \backslash \mathrm{H}, 2.0938083814,0.4068391728,0.0550888479 \backslash \mathrm{H},-1.2835162888,-1.32438429$ $17,0.282238691 \backslash \mathrm{H},-2.2612433715,0.1456435331,-0.2742872801 \backslash \mathrm{H},-0.0815312$ $686,1.1245362404,0.9899877541 \backslash \mathrm{H},-0.1027629258,1.3124119252,-0.73792174$ $01 \backslash \mathrm{H}, 1.2980411543,-0.7761812214,-0.9857012577 \backslash \mathrm{H}, 1.2801217706,-0.987445$

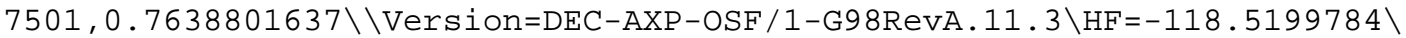
$\mathrm{S} 2=0.75394 \backslash \mathrm{S} 2-1=0 . \backslash \mathrm{S} 2 \mathrm{~A}=0.75001 \backslash \mathrm{RMSD}=3.100 \mathrm{e}-09 \backslash \mathrm{RMSF}=3.596 \mathrm{e}-06 \backslash \mathrm{Dipole}=0$. $0709948,0.0891893,0.0423908 \backslash \mathrm{PG}=\mathrm{C} 01 \quad[\mathrm{X}(\mathrm{C} 3 \mathrm{H} 7)] \backslash \backslash @$

\section{UB3LYP / 6-311+G (3df, 2p)}

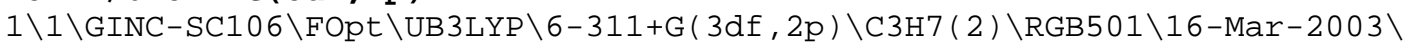
$0 \backslash \backslash \#$ B3LYP/6-311+G(3DF,2P) SCF=TIGHT OPT=TIGHT FREQ MAXDISK=262144000

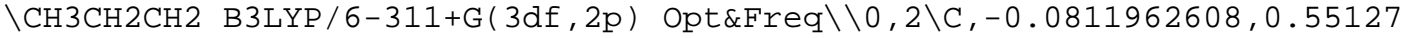


$77283,0.0446518988 \backslash \mathrm{C},-1.3017351697,-0.2927624029,-0.0259479114 \backslash \mathrm{C}, 1.225$ $6270085,-0.2422479219,-0.0333426201 \backslash \mathrm{H}, 2.0926697485,0.4137533063,0.0519$ $538481 \backslash \mathrm{H},-1.2758876706,-1.332120898,0.272557334 \backslash \mathrm{H},-2.2647503201,0.1384$ $830938,-0.2605820412 \backslash \mathrm{H},-0.0858898312,1.1327744237,0.9807305596 \backslash \mathrm{H},-0.10$ $75718399,1.3064902297,-0.7484562229 \backslash \mathrm{H}, 1.3014493066,-0.77946219,-0.9797$ $696349 \backslash \mathrm{H}, 1.2838071382,-0.9775223862,0.771397954 \backslash \backslash$ Version=DEC-AXP $-O S F / 1$ $-G 98 R e v A .11 .3 \backslash \mathrm{HF}=-118.5166777 \backslash \mathrm{S} 2=0.753967 \backslash \mathrm{S} 2-1=0 . \backslash \mathrm{S} 2 \mathrm{~A}=0.75001 \backslash \mathrm{RMSD}=9.0$ $48 e-09 \backslash \mathrm{RMSF}=3.386 \mathrm{e}-06 \backslash \mathrm{Dipole}=0.087157,0.1021474,0.0432445 \backslash \mathrm{PG}=\mathrm{C} 01 \quad[\mathrm{X}(\mathrm{C} 3$ H7) $] \backslash \backslash @$

\section{UMPW1K/6-31+G (d, p)}

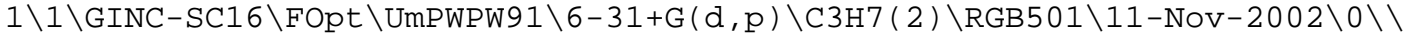
\#MPWPW91 6-31+G** IOP (5/45=10000428) IOP (5/46=05720572) IOP (5/47=10001

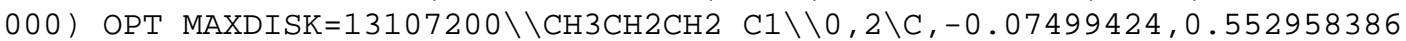
$6,0.0483251784 \backslash \mathrm{C},-1.2950736347,-0.2872398445,-0.0279466351 \backslash \mathrm{C}, 1.2146223$ $064,-0.2473278583,-0.0361524942 \backslash \mathrm{H}, 2.0873120971,0.3976995266,0.04588325$ $23 \backslash \mathrm{H},-1.2734291237,-1.3196947848,0.2881865303 \backslash \mathrm{H},-2.2542311067,0.146177$ $6153,-0.2657473962 \backslash \mathrm{H},-0.0779407024,1.1249725602,0.9851465496 \backslash \mathrm{H},-0.0977$ $413347,1.3060835511,-0.742362282 \backslash \mathrm{H}, 1.2798488614,-0.7825838509,-0.98184$ $96946 \backslash \mathrm{H}, 1.2688547185,-0.9829987209,0.7653867462 \backslash \backslash$ Version=DEC-AXP $-O S F / 1$ $-G 98 R e v A .11 .3 \backslash \mathrm{HF}=-118.4569029 \backslash \mathrm{S} 2=0.755506 \backslash \mathrm{S} 2-1=0 . \backslash \mathrm{S} 2 \mathrm{~A}=0.750019 \backslash \mathrm{RMSD}=5$. $892 e-09 \backslash \mathrm{RMSF}=3.022 e-06 \backslash \mathrm{Dipole}=0.0717866,0.0903279,0.0525449 \backslash \mathrm{PG}=\mathrm{C} 01] \mathrm{X}($ $\mathrm{C} 3 \mathrm{H} 7$ ) $] \backslash \backslash \mathrm{e}$

\section{UMP 2/6-31G (d)}

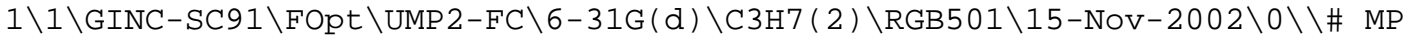
2/6-31G* FOPT FREQ MAXDISK=262144000 \\CH2CH2 MP2/6-31G(d) Opt\&Freq \\0, $2 \backslash \mathrm{C},-0.259768023,-0.5028442094,0.0000229782 \backslash \mathrm{C}, 1.2320743547,-0.50068430$ $67,0.0309469992 \backslash \mathrm{C},-0.8538473948,0.905179113,-0.0528529987 \backslash \mathrm{H},-1.9469854$ $837,0.8736417236,-0.0575903481 \backslash \mathrm{H}, 1.763632808,0.2781246032,0.5655489067$ $\backslash \mathrm{H}, 1.7977684855,-1.3753970293,-0.265571137 \backslash \mathrm{H},-0.6558392452,-1.02353441$ $32,0.8871202942 \backslash \mathrm{H},-0.6090334403,-1.0851676532,-0.8608593265 \backslash \mathrm{H},-0.52300$ $02837,1.4309494661,-0.9523468892 \backslash \mathrm{H},-0.5372964621,1.4914797216,0.814996$ $6281 \backslash \backslash$ Version=DEC-AXP-OSF $/ 1-G 98$ RevA. $11.3 \backslash \mathrm{HF}=-117.6313287 \backslash \mathrm{MP} 2=-118.0004$ $657 \backslash \mathrm{PUHF}=-117.6345747 \backslash \mathrm{PMP} 2-0=-118.0025633 \backslash \mathrm{S} 2=0.762603 \backslash \mathrm{S} 2-1=0.75364 \backslash \mathrm{S} 2 \mathrm{~A}$ $=0.750107 \backslash \mathrm{RMSD}=7.053 \mathrm{e}-09 \backslash \mathrm{RMSF}=7.993 \mathrm{e}-06 \backslash \mathrm{Dipole}=-0.068883,-0.0410691,0$. $0524296 \backslash P G=C 01 \quad[X(C 3 H 7)] \backslash \backslash @$

\section{UQCISD/6-31G (d)}

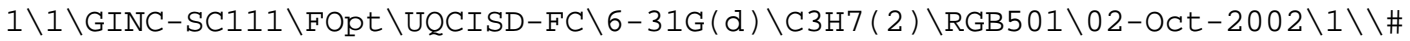
$\mathrm{N}$ QCISD 6-31G* FOPT=(Z-MATRIX) FREQ TEST MAXDISK=196608000 \\CH3CH2CH2 Opt\&Freq $\mathrm{C} 1 \backslash \backslash 0,2 \backslash \mathrm{C} \backslash \mathrm{C}, 1, \mathrm{~B} 1 \backslash \mathrm{C}, 1, \mathrm{~B} 2,2, \mathrm{~A} 1 \backslash \mathrm{H}, 3, \mathrm{~B} 3,1, \mathrm{~A} 2,2, \mathrm{D} 1,0 \backslash \mathrm{H}, 2, \mathrm{~B} 4,1, \mathrm{~A} 3,3$

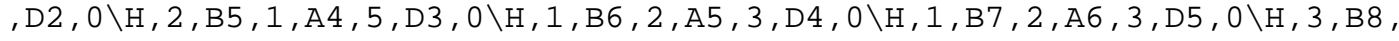
$1, \mathrm{~A} 7,4, \mathrm{D} 6,0 \backslash \mathrm{H}, 3, \mathrm{~B} 9,1, \mathrm{~A} 8,4, \mathrm{D} 7,0 \backslash \backslash \mathrm{B} 1=1.49677 \backslash \mathrm{B} 2=1.53245 \backslash \mathrm{B} 3=1.09704 \backslash \mathrm{B} 4=1$. $08806 \backslash B 5=1.08714 \backslash B 6=1.10595 \backslash B 7=1.10014 \backslash B 8=1.09684 \backslash B 9=1.09776 \backslash A 1=112.94$ $5 \backslash A 2=111.2185 \backslash A 3=120.3212 \backslash A 4=121.0711 \backslash A 5=110.0717 \backslash A 6=109.5683 \backslash A 7=110.7$ $855 \backslash A 8=110.7751 \backslash D 1=-179.0186 \backslash D 2=33.7141 \backslash D 3=167.2795 \backslash D 4=-121.8955 \backslash D 5=12$ $2.7068 \backslash \mathrm{D} 6=-120.3609 \backslash \mathrm{D} 7=120.005 \backslash \backslash$ Version=DEC-AXP-OSF $/ 1-\mathrm{G} 98 \mathrm{RevA} .11 .3 \backslash \mathrm{HF}=$ $-117.6309789 \backslash \mathrm{MP} 2=-118.0003702 \backslash \mathrm{MP} 3=-118.0363538 \backslash \mathrm{MP} 4 \mathrm{D}=-118.0464716 \backslash \mathrm{MP} 4 \mathrm{DQ}$ $=-118.040796 \backslash \mathrm{PUHF}=-117.6342789 \backslash \mathrm{PMP} 2-0=-118.0025108 \backslash \mathrm{PMP} 3-0=-118.0376486$ $\backslash \mathrm{MP} 4 \mathrm{SDQ}=-118.0435427 \backslash \mathrm{QCISD}=-118.0459761 \backslash \mathrm{S} 2=0.762946 \backslash \mathrm{S} 2-1=0.753801 \backslash \mathrm{S} 2 \mathrm{~A}=$ $0.750113 \backslash \mathrm{RMSD}=2.540 \mathrm{e}-09 \backslash \mathrm{RMSF}=1.461 \mathrm{e}-06 \backslash \mathrm{Dipole}=-0.0375858,0.0449901,-0$. $0697833 \backslash \mathrm{PG}=\mathrm{C} 01 \quad[\mathrm{X}(\mathrm{C} 3 \mathrm{H} 7)] \backslash \backslash @$

\section{$\operatorname{UCCSD}(\mathrm{T}) / 6-311 \mathrm{G}(\mathrm{d}, \mathrm{p})$}

$1 \backslash 1 \backslash G I N C-S C 124 \backslash F O p t \backslash U C C S D(T)-F C \backslash 6-311 G(d, p) \backslash C 3 H 7(2) \backslash R G B 501 \backslash 03-D e C-2002$ $\backslash 1 \backslash \backslash \# \mathrm{~N} \operatorname{CCSD}(\mathrm{T}) / 6-311 \mathrm{G} * * \mathrm{OPT}=(\mathrm{Z}-\mathrm{MATRIX})$ FREQ TEST MAXDISK=262144000 $\backslash \mathrm{CH}$ 3CH2CH2 Opt\&Freq $\mathrm{C} 1 \backslash \backslash 0,2 \backslash \mathrm{C} \backslash \mathrm{C}, 1, \mathrm{~B} 1 \backslash \mathrm{C}, 1, \mathrm{~B} 2,2, \mathrm{~A} 1 \backslash \mathrm{H}, 3, \mathrm{~B} 3,1, \mathrm{~A} 2,2, \mathrm{D} 1,0 \backslash \mathrm{H}, 2, \mathrm{~B}$ $4,1, \mathrm{~A} 3,3, \mathrm{D} 2, \mathrm{O} \backslash \mathrm{H}, 2, \mathrm{~B} 5,1, \mathrm{~A} 4,5, \mathrm{D} 3,0 \backslash \mathrm{H}, 1, \mathrm{~B} 6,2, \mathrm{~A} 5,3, \mathrm{D} 4,0 \backslash \mathrm{H}, 1, \mathrm{~B} 7,2, \mathrm{~A} 6,3, \mathrm{D} 5,0$ 
$\backslash \mathrm{H}, 3, \mathrm{~B} 8,1, \mathrm{~A} 7,4, \mathrm{D} 6,0 \backslash \mathrm{H}, 3, \mathrm{~B} 9,1, \mathrm{~A} 8,4, \mathrm{D} 7,0 \backslash \backslash \mathrm{B} 1=1.5009599 \backslash \mathrm{B} 2=1.5375406 \backslash \mathrm{B} 3=1$ $.09733048 \backslash B 4=1.08864158 \backslash B 5=1.08740505 \backslash B 6=1.10616045 \backslash B 7=1.10003749 \backslash B 8=1$ $.09731795 \backslash B 9=1.09820335 \backslash A 1=112.78421522 \backslash A 2=111.15023139 \backslash A 3=119.9279220$ $8 \backslash A 4=120.94699633 \backslash A 5=109.86102615 \backslash A 6=109.59611088 \backslash A 7=110.56554282 \backslash A 8=1$ $10.53747716 \backslash \mathrm{D} 1=-179.15563531 \backslash \mathrm{D} 2=34.86905343 \backslash \mathrm{D} 3=166.7989188 \backslash \mathrm{D} 4=-121.527$ $58812 \backslash D 5=122.69937159 \backslash D 6=-120.40227658 \backslash D 7=120.09385477 \backslash \backslash$ Version=DEC $-A X$ $\mathrm{P}-\mathrm{OSF} / 1-\mathrm{G} 98 \mathrm{RevA} .11 .3 \backslash \mathrm{HF}=-117.6629391 \backslash \mathrm{MP} 2=-118.0973775 \backslash \mathrm{MP} 3=-118.138057 \backslash$ $M P 4 D=-118.149429 \backslash M P 4 D Q=-118.1410525 \backslash P U H F=-117.6662393 \backslash P M P 2-0=-118.0995$ $048 \backslash \mathrm{PMP} 3-0=-118.1393166 \backslash \mathrm{MP} 4 \mathrm{SDQ}=-118.1436791 \backslash \mathrm{CCSD}=-118.1446339 \backslash \mathrm{CCSD}(\mathrm{T})=$ $-118.1591261 \backslash \mathrm{S} 2=0.763033 \backslash \mathrm{S} 2-1=0.753721 \backslash \mathrm{S} 2 \mathrm{~A}=0.750114 \backslash \mathrm{RMSD}=5.193 e-09 \backslash \mathrm{RMS}$ $\mathrm{F}=2.501 \mathrm{e}-05 \backslash \mathrm{PG}=\mathrm{C} 01 \quad[\mathrm{X}(\mathrm{C} 3 \mathrm{H} 7)] \backslash \backslash @$

\section{$\mathrm{CH} \equiv \mathrm{CH}$}

\section{RHF /6-31G (d)}

$1 \backslash 1 \backslash G I N C-S C 75 \backslash F O p t \backslash R H F \backslash 6-31 G(d) \backslash C 2 H 2 \backslash R G B 501 \backslash 22-A u g-2002 \backslash 0 \backslash \backslash \# N$ RHF 6-31 G* SCF $=$ TIGHT OPT=TIGHT FREQ MAXDISK=13107200\\Acetylene $\backslash \backslash 0,1 \backslash C, 0 ., 0 .,-$ $0.5927229054 \backslash \mathrm{C}, 0 ., 0 \ldots, 0.5927229054 \backslash \mathrm{H}, 0 ., 0 \ldots,-1.6496755002 \backslash \mathrm{H}, 0 ., 0 ., 1.6496$ $755002 \backslash \backslash$ Version=DEC-AXP-OSE /1-G98RevA.11.3 State=1-SGG \HF=-76.8178265 $R M S D=4.239 e-09 \backslash R M S F=1.015 e-08 \backslash D i p o l e=0 ., 0 ., 0 . \backslash P G=D * H \quad[C *(H 1 C 1 . C 1 H 1)] \backslash \backslash$ c

\section{RB3LYP / 6-31G (d)}

$1 \backslash 1 \backslash G I N C-S C 116 \backslash F O p t \backslash R B 3 L Y P \backslash 6-31 G(d) \backslash C 2 H 2 \backslash R G B 501 \backslash 22-A u g-2002 \backslash 0 \backslash \backslash \# N$ B3LY P 6-31G* SCF=TIGHT OPT=TIGHT FREQ MAXDISK=13107200\\Acetylene Opt\&Freq

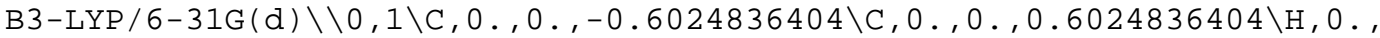
$0 .,-1.6691266823 \backslash \mathrm{H}, 0 ., 0 ., 1.6691266823 \backslash \backslash$ Version=DEC-AXP-OSF / 1-G98RevA. 1 1.3 $\backslash$ State $=1-S G G \backslash H F=-77.3256462 \backslash R M S D=2.872 e-10 \backslash R M S F=1.007 e-07 \backslash D i p o l e=0$. , $0 ., 0 . \backslash \mathrm{PG}=\mathrm{D} * \mathrm{H} \quad[\mathrm{C} *(\mathrm{H} 1 \mathrm{C} 1 . \mathrm{C} 1 \mathrm{H} 1)] \backslash \backslash \mathrm{Q}$

\section{RB3LYP / 6-31G (2df, p)}

$1 \backslash 1 \backslash G I N C-S C 160 \backslash F O p t \backslash R B 3 L Y P \backslash 6-31 G(2 d f, p) \backslash C 2 H 2 \backslash R G B 501 \backslash 05-M a y-2002 \backslash 0 \backslash \backslash \# N$ B3LYP 6-31G(2DF,P) TEST SCF=DIRECT OPT FREQ MAXDISK=117964800\\Acetyle

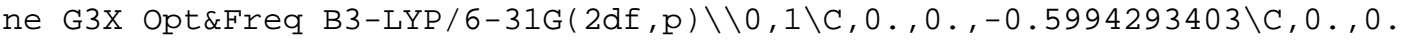
$, 0.5994293403 \backslash \mathrm{H}, 0 ., 0 .,-1.6618455204 \backslash \mathrm{H}, 0 ., 0 ., 1.6618455204 \backslash \backslash$ Version=DEC$\mathrm{AXP}-\mathrm{OSF} / 1-\mathrm{G} 98 \mathrm{RevA} .11 .3 \backslash \mathrm{HF}=-77.335268 \backslash \mathrm{RMSD}=3.709 \mathrm{e}-09 \backslash \mathrm{RMSF}=1.425 \mathrm{e}-04 \backslash \mathrm{Dip}$ $\mathrm{ole}=0 ., 0 ., 0 . \backslash \mathrm{PG}=\mathrm{D} * \mathrm{H} \quad[\mathrm{C} *(\mathrm{H} 1 \mathrm{C} 1 . \mathrm{C} 1 \mathrm{H} 1)] \backslash \backslash \mathrm{e}$

\section{RB3LYP/CC-PVTZ}

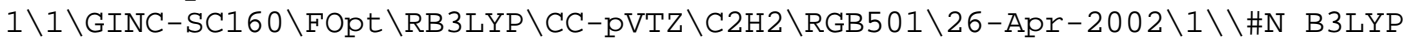
CC-PVTZ TEST SCF=DIRECT OPT=(Z-MATRIX, TIGHT) FREQ MAXDISK=13107200\\A

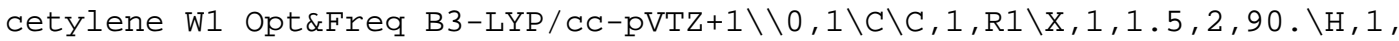
$\mathrm{R} 2,3,90 ., 2,180 ., 0 \backslash \mathrm{X}, 2,1.5,1,90 ., 3,180 ., 0 \backslash \mathrm{H}, 2, \mathrm{R} 2,5,90 ., 1,180 ., 0 \backslash \backslash \mathrm{R} 1=1.1$ $9602563 \backslash \mathrm{R} 2=1.06160466 \backslash \backslash$ Version=DEC-AXP-OSF/1-G98RevA.11.3\State=1-SGG $\mathrm{HF}=-77.3635521 \backslash \mathrm{RMSD}=3.693 e-09 \backslash \mathrm{RMSF}=1.612 e-07 \backslash \mathrm{Dipole}=0 ., 0 ., 0 . \backslash \mathrm{PG}=\mathrm{D} * \mathrm{H} \quad[\mathrm{C}$ * ( $\mathrm{H} 1 \mathrm{C} 1 . \mathrm{C} 1 \mathrm{H} 1)] \backslash \backslash \mathrm{C}$

\section{RB3LYP/6-311+G (3df, 2p)}

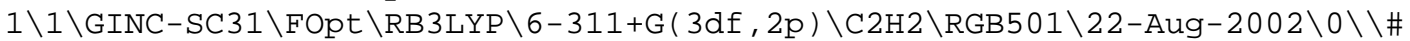

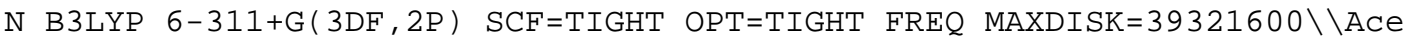

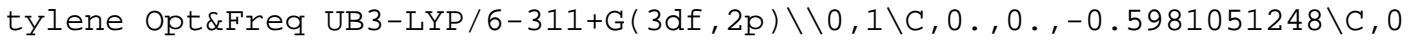
, $0 ., 0.5981051248 \backslash \mathrm{H}, 0 ., 0 .,-1.6602910697 \backslash \mathrm{H}, 0 ., 0 ., 1.6602910697 \backslash \backslash$ Version $=$ $\mathrm{DEC}-\mathrm{AXP}-\mathrm{OSF} / 1-\mathrm{G} 98 \mathrm{RevA} .11 .3 \backslash \mathrm{State}=1-\mathrm{SGG} \backslash \mathrm{HF}=-77.3619986 \backslash \mathrm{RMSD}=2.447 \mathrm{e}-09 \backslash \mathrm{R}$ $\mathrm{MSF}=5.450 \mathrm{e}-06 \backslash \mathrm{Dipole}=0 ., 0 ., 0 . \backslash \mathrm{PG}=\mathrm{D} * \mathrm{H} \quad[\mathrm{C} *(\mathrm{H} 1 \mathrm{C} 1 . \mathrm{C} 1 \mathrm{H} 1)] \backslash \backslash @$

\section{RMPW1K/6-31+G (d, p)}

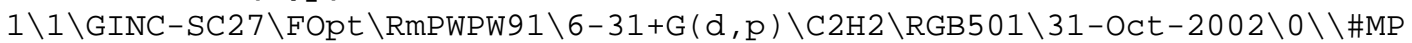
WPW91 6-31+G** IOP $(5 / 45=10000428) \quad \operatorname{IOP}(5 / 46=05720572) \quad \operatorname{IOP}(5 / 47=10001000$ ) OPT MAXDISK=13107200\\Acetylene $\backslash \backslash 0,1 \backslash \mathrm{C}, 0 ., 0 \ldots, 0.599403245 \backslash \mathrm{C}, 0 ., 0 ., 0$. $599403245 \backslash \mathrm{H}, 0.0 .,-1.6609447805 \backslash \mathrm{H}, 0 ., 0 ., 1.6609447805 \backslash \backslash$ Version=DEC-AXP - 
OSF /1-G98RevA.11.3\State=1-SGG $\backslash \mathrm{HF}=-77.3017259 \backslash \mathrm{RMSD}=2.473 \mathrm{e}-09 \backslash \mathrm{RMSF}=1.54$ $9 e-05 \backslash \mathrm{Dipole}=0 ., 0 ., 0 . \backslash \mathrm{PG}=\mathrm{D} * \mathrm{H} \quad[\mathrm{C} *(\mathrm{H} 1 \mathrm{C} 1 . \mathrm{C} 1 \mathrm{H} 1)] \backslash \backslash \mathrm{C}$

\section{RMP 2/6-31G (d)}

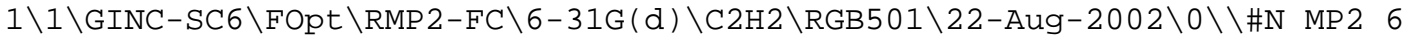

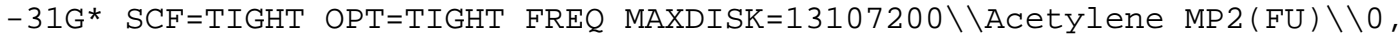
$1 \backslash \mathrm{C}, 0.0 \ldots,-0.6088286179 \backslash \mathrm{C}, 0 ., 0 ., 0.6088286179 \backslash \mathrm{H}, 0.0 \ldots,-1.6750163708 \backslash \mathrm{H}, 0$ ., $0.1 .6750163708 \backslash \backslash$ Version=DEC-AXP-OSF $/ 1-G 98$ RevA.11.3 State $=1-$ SGG $\backslash \mathrm{HF}=-$ $76.8153928 \backslash \mathrm{MP} 2=-77.0667935 \backslash \mathrm{RMSD}=4.344 \mathrm{e}-09 \backslash \mathrm{RMSF}=7.655 \mathrm{e}-07 \backslash \mathrm{Dipole}=0 ., 0 .$, $0 . \backslash P G=D * H \quad[C *(\mathrm{H} 1 \mathrm{C} 1 . \mathrm{C} 1 \mathrm{H} 1)] \backslash \backslash @$

\section{RQCISD/6-31G (d)}

$1 \backslash 1 \backslash G I N C-S C 111 \backslash F O p t \backslash R Q C I S D-F C \backslash 6-31 G(d) \backslash C 2 H 2 \backslash R G B 501 \backslash 19-S e p-2002 \backslash 1 \backslash \backslash \# N Q$

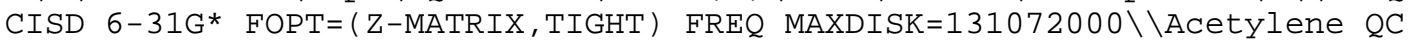
$\mathrm{ISD} / 6-31 \mathrm{G} \star \backslash \backslash 0,1 \backslash \mathrm{C} \backslash \mathrm{C}, 1, \mathrm{R} 1 \backslash \mathrm{X}, 1,1.5,2,90 . \backslash \mathrm{H}, 1, \mathrm{R} 2,3,90 ., 2,180 ., 0 \backslash \mathrm{X}, 2,1.5,1$ $, 90 ., 3,180 ., 0 \backslash H, 2, R 2,5,90 ., 1,180 ., 0 \backslash \backslash R 1=1.2125989 \backslash R 2=1.06883869 \backslash \backslash$ Versi on=DEC-AXP $-O S F / 1-G 98 R e v A .11 .3 \backslash$ State $=1-S G G \backslash H F=-76.815957 \backslash \mathrm{MP} 2=-77.066734$ $4 \backslash \mathrm{MP} 3=-77.0760026 \backslash \mathrm{MP} 4 \mathrm{D}=-77.0832733 \backslash \mathrm{MP} 4 \mathrm{DQ}=-77.0777937 \backslash \mathrm{MP} 4 \mathrm{SDQ}=-77.081609$ $7 \backslash \mathrm{QCISD}=-77.0834463 \backslash \mathrm{RMSD}=2.182 \mathrm{e}-09 \backslash \mathrm{RMSF}=1.276 \mathrm{e}-07 \backslash \mathrm{D}$ pole=0, $0 ., 0 . \backslash \mathrm{PG}=\mathrm{D}$ $\star_{\mathrm{H}}[\mathrm{C} *(\mathrm{H} 1 \mathrm{C} 1 . \mathrm{C} 1 \mathrm{H} 1)] \backslash \backslash \mathrm{Q}$

\section{$\operatorname{RCCSD}(T) / 6-311 G(d, p)$}

$1 \backslash 1 \backslash G I N C-S C 12 \backslash F O p t \backslash R C C S D(T)-F C \backslash 6-311 G(d, p) \backslash C 2 H 2 \backslash R G B 501 \backslash 19-S e p-2002 \backslash 1 \backslash \backslash$

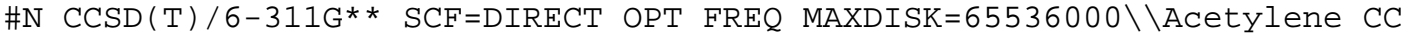
$\mathrm{SD}(\mathrm{T}) / 6-311 \mathrm{G} * \star \backslash \backslash 0,1 \backslash \mathrm{C} \backslash \mathrm{C}, 1, \mathrm{R} 1 \backslash \mathrm{X}, 1,1.5,2,90 . \backslash \mathrm{H}, 1, \mathrm{R} 2,3,90 ., 2,180 ., 0 \backslash \mathrm{X}, 2,1$ $.5,1,90 ., 3,180 ., 0 \backslash \mathrm{H}, 2, \mathrm{R} 2,5,90 ., 1,180 ., 0 \backslash \backslash \mathrm{R} 1=1.21455367 \backslash \mathrm{R} 2=1.06733362 \backslash \backslash$ Version=DEC-AXP-OSF $/ 1-G 98$ RevA. $11.3 \backslash$ State $=1-S G G \backslash H F=-76.8387905 \backslash M P 2=-77$. $1112294 \backslash \mathrm{MP} 3=-77.120507 \backslash \mathrm{MP} 4 \mathrm{D}=-77.128394 \backslash \mathrm{MP} 4 \mathrm{DQ}=-77.1216232 \backslash \mathrm{MP} 4 \mathrm{SDQ}=-77.12$ $55581 \backslash \mathrm{CCSD}=-77.125982 \backslash \mathrm{CCSD}(\mathrm{T})=-77.1393216 \backslash \mathrm{RMSD}=1.084 \mathrm{e}-09 \backslash \mathrm{RMSF}=3.092 \mathrm{e}-0$ $5 \backslash P G=D * H \quad[C *(H 1 C 1 . C 1 H 1)] \backslash \backslash @$

\section{$\cdot \mathrm{CH} 3--\mathrm{CH} \equiv \mathrm{CH}$}

\section{$\mathrm{UHF} / 6-31 \mathrm{G}(\mathrm{d})$}

$1 \backslash 1 \backslash G I N C-S C 122 \backslash F T S \backslash U H F \backslash 6-31 G(d) \backslash C 3 H 5(2) \backslash R G B 501 \backslash 26-A u g-2002 \backslash 0 \backslash \backslash \# N \quad U H F \quad 6$ $-31 \mathrm{G} * \mathrm{SCF}=\mathrm{TIGHT}$ OPT $=(\mathrm{TS}, \mathrm{CALCFC}, \mathrm{NOEIGENTEST}, \mathrm{TIGHT}) \quad$ FREQ MAXDISK=1310720 $0 \backslash \backslash \mathrm{TS} \backslash \backslash 0,2 \backslash \mathrm{C},-0.0917278051,0 \ldots,-1.6017717368 \backslash \mathrm{C},-0.4471924907,0 ., 0.69776$ $24904 \backslash \mathrm{C}, 0.5580033949,0 ., 1.4219033744 \backslash \mathrm{H}, 1.5923748098,0 ., 1.6539981448 \backslash \mathrm{H}$, $-1.5060977521,0 ., 0.6242250132 \backslash \mathrm{H}, 0.9808879986,0 .,-1.6623221243 \backslash \mathrm{H},-0.590$ $8318254,0.916671393,-1.861632901 \backslash \mathrm{H},-0.5908318254,-0.916671393,-1.86163$ $2901 \backslash \backslash$ Version=DEC-AXP-OSF /1-G98RevA.11.3 State $=2-A^{\prime} \backslash \mathrm{HF}=-116.3572837 \backslash \mathrm{S} 2$ $=1.15882 \backslash \mathrm{S} 2-1=0 . \backslash \mathrm{S} 2 \mathrm{~A}=0.856976 \backslash \mathrm{RMSD}=8.928 \mathrm{e}-09 \backslash \mathrm{RMSF}=1.586 \mathrm{e}-06 \backslash \mathrm{Dipole}=0.0$ $279767,0 .,-0.1417213 \backslash \mathrm{PG}=\mathrm{CS} \quad[\mathrm{SG}(\mathrm{C} 3 \mathrm{H} 3), \mathrm{X}(\mathrm{H} 2)] \backslash \backslash \mathrm{C}$

\section{UB3LYP / 6-31G (d)}

$1 \backslash 1 \backslash G I N C-S C 111 \backslash F T S \backslash U B 3 L Y P \backslash 6-31 G(d) \backslash C 3 H 5(2) \backslash R G B 501 \backslash 20-A u g-2002 \backslash 0 \backslash \backslash \# N$ UB 3LYP 6-31G* SCF=TIGHT OPT=(TS, CALCFC, NOEIGENTEST, TIGHT) INT=ULTRAFINE FREQ MAXDISK=26214400\\TS CH3+CHCH Rotational potencial Opt and Freq $\backslash \backslash$ $0,2 \backslash \mathrm{C},-0.3596996685,0 .,-1.5754719249 \backslash \mathrm{C},-0.3226972741,0 ., 0.7824166815 \backslash \mathrm{C}$ , $0.7909204591,0 ., 1.2886702837 \backslash \mathrm{H}, 1.8386353962,0 ., 1.4926366479 \backslash \mathrm{H},-1.3920$ $209695,0 ., 0.7846717887 \backslash \mathrm{H}, 0.7019929411,0 .,-1.7916095522 \backslash \mathrm{H},-0.8998742334$ $, 0.9285750575,-1.7296945631 \backslash \mathrm{H},-0.8998742334,-0.9285750575,-1.729694563$ $1 \backslash \backslash$ Version=DEC-AXP-OSF /1-G98RevA.11.3 State=2-A' \HF=-117.1555028 \S2=0. $771723 \backslash \mathrm{S} 2-1=0 . \backslash \mathrm{S} 2 \mathrm{~A}=0.750175 \backslash \mathrm{RMSD}=5.717 \mathrm{e}-09 \backslash \mathrm{RMSF}=3.516 \mathrm{e}-07 \backslash \mathrm{Dipole}=-0.00$ $43923,0 .,-0.0743853 \backslash P G=C S \quad[S G(C 3 H 3), X(H 2)] \backslash \backslash @$

\section{UB3LYP / 6-31G (2df, p)}

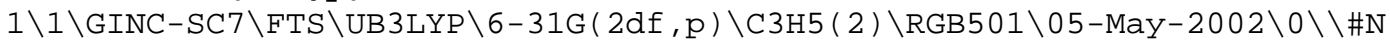
UB3LYP 6-31G(2DF,P) TEST SCF=DIRECT OPT=(TS, CALCFC, NOEIGENTEST) FREQ M AXDISK=117964800 \TS Opt\&Freq UB3LYP/6-31G $(2 \mathrm{df}, \mathrm{p}) \backslash \backslash 0,2 \backslash \mathrm{C}, 0.9006516301$, 
$-1.315302789,0 . \backslash \mathrm{C}, 0.0181996294,0.8357660356,0 . \backslash \mathrm{C},-1.1989145029,0.89497$ $75775,0 . \backslash \mathrm{H},-2.2471163693,0.7163820392,0 . \backslash \mathrm{H}, 1.0096590785,1.226348971,0$. $\backslash \mathrm{H},-0.0026689392,-1.9112491348,0 . \backslash \mathrm{H}, 1.4602528454,-1.26206341,0.9267575$ $647 \backslash \mathrm{H}, 1.4602528454,-1.26206341,-0.9267575647 \backslash \backslash$ Version=DEC-AXP - OSF $/ 1-G 9$ 8RevA.11.3 State $=2-A^{\prime} \backslash H F=-117.1696098 \backslash S 2=0.771883 \backslash S 2-1=0 . \backslash S 2 A=0.750174$ $\backslash \mathrm{RMSD}=1.749 \mathrm{e}-09 \backslash \mathrm{RMSF}=4.052 \mathrm{e}-05 \backslash \mathrm{Dipole}=0.0263685,-0.0593606,0 . \backslash \mathrm{PG}=\mathrm{CS} \quad[\mathrm{S}$ $\mathrm{G}(\mathrm{C} 3 \mathrm{H} 3), \mathrm{X}(\mathrm{H} 2)] \backslash \backslash \mathrm{C}$

\section{UB3LYP /CC-PVTZ}

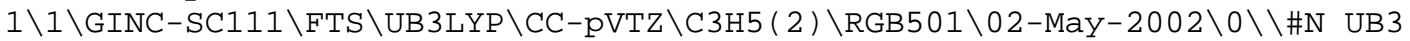
LYP CC-PVTZ TEST SCF=DIRECT OPT=(TS, CALCFC, NOEIGENTEST) FREQ MAXDISK=1

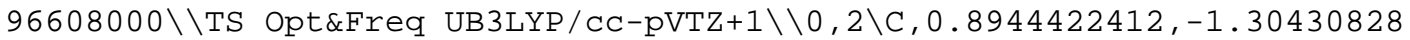
$63,0 . \backslash \mathrm{C}, 0.0217472771,0.8251628441,0 . \backslash \mathrm{C},-1.1929608918,0.8872162968,0 . \backslash \mathrm{H}$ $,-2.2420776471,0.7192354149,0 . \backslash \mathrm{H}, 1.0077032355,1.2275442522,0 . \backslash \mathrm{H},-0.006$ $6482947,-1.8972464823,0 . \backslash \mathrm{H}, 1.4508254736,-1.2489791561,0.9244351106 \backslash \mathrm{H}, 1$ $.4508254736,-1.2489791561,-0.9244351106 \backslash \backslash$ Version=DEC-AXP-OSF /1-G98RevA $.11 .3 \backslash$ State $=2-A^{\prime} \backslash \mathrm{HF}=-117.2104672 \backslash \mathrm{S} 2=0.770945 \backslash \mathrm{S} 2-1=0 . \backslash \mathrm{S} 2 \mathrm{~A}=0.750156 \backslash \mathrm{RMSD}$ $=2.609 e-09 \backslash \mathrm{RMSF}=8.461 e-06 \backslash \mathrm{Dipole}=0.0144798,-0.0550922,0 . \backslash \mathrm{PG}=\mathrm{CS} \quad[\mathrm{SG}(\mathrm{C} 3 \mathrm{H}$ 3), $\mathrm{X}(\mathrm{H} 2)] \backslash \backslash \mathrm{C}$

\section{UB3LYP / 6-311+G (3df, 2p)}

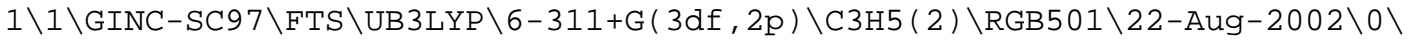
$\backslash \# N$ UB3LYP 6-311+G(3DF, 2P) TEST SCF=TIGHT OPT=(TS, CALCFC, NOEIGENTEST, T IGHT) FREQ MAXDISK=39321600 \TS Opt\&Freq UB3LYP/6-311+G(3df,2p) tight $\backslash 0,2 \backslash \mathrm{C}, 0.9140302476,-1.2888102578,0 . \backslash \mathrm{C}, 0.0097063454,0.8257619149,0 . \backslash \mathrm{C}$, $-1.2060923242,0.865398971,0 . \backslash \mathrm{H},-2.2526457911,0.6793239672,0 . \backslash \mathrm{H}, 0.98856$ $84046,1.2461921773,0 . \backslash \mathrm{H}, 0.021218567,-1.8939390016,0 . \backslash \mathrm{H}, 1.4684966037,-1$ $.2228404556,0.9247119948 \backslash \mathrm{H}, 1.4684966037,-1.2228404556,-0.9247119948 \backslash \backslash \mathrm{V}$ ersion=DEC-AXP-OSF /1-G98RevA.11.3\State=2-A ' \HF=-117.2077155 \S2=0.7706 $77 \backslash S 2-1=0 . \backslash S 2 A=0.750152 \backslash R M S D=9.702 e-09 \backslash R M S F=5.949 e-07 \backslash D i p o l e=0.0123962$ $,-0.0511266,0 . \backslash \mathrm{PG}=\mathrm{CS}[\mathrm{SG}(\mathrm{C} 3 \mathrm{H} 3), \mathrm{X}(\mathrm{H} 2)] \backslash \backslash @$

\section{UMPW1K/6-31+G (d, p)}

$1 \backslash 1 \backslash G I N C-S C 48 \backslash F T S \backslash U m P W P W 91 \backslash 6-31+G(d, p) \backslash C 3 H 5(2) \backslash R G B 501 \backslash 01-N o v-2002 \backslash 0 \backslash \backslash \#$ MPWPW91 6-31+G** IOP $(5 / 45=10000428) \quad$ IOP $(5 / 46=05720572) \quad$ IOP $(5 / 47=100010$ $00) \mathrm{MAXDISK}=39321600 \mathrm{OPT}=(\mathrm{TS}, \mathrm{CALCFC}, \mathrm{NOEIGENTEST}) \backslash \backslash \mathrm{tS} \backslash \backslash 0,2 \backslash \mathrm{C}, 0.91408157$ $08,-1.287446357,0 . \backslash \mathrm{C}, 0.0107408092,0.8295718983,0 . \backslash \mathrm{C},-1.2068835294,0.85$ $95283472,0 . \backslash \mathrm{H},-2.2530128978,0.6774665865,0 . \backslash \mathrm{H}, 0.9941782166,1.236549693$ , $0 . \backslash \mathrm{H}, 0.0196601346,-1.8874548532,0 . \backslash \mathrm{H}, 1.4657707214,-1.2182423785,0.924$ $3797797 \backslash \mathrm{H}, 1.4657707214,-1.2182423785,-0.9243797797 \backslash \backslash$ Version=DEC-AXP $-O S$ $\mathrm{F} / 1-\mathrm{G} 98 \mathrm{RevA} .11 .3 \backslash$ State $=2-\mathrm{A}^{\prime} \backslash \mathrm{HF}=-117.1246295 \backslash \mathrm{S} 2=0.795736 \backslash \mathrm{S} 2-1=0 . \backslash \mathrm{S} 2 \mathrm{~A}=0$. $750704 \backslash \mathrm{RMSD}=3.211 \mathrm{e}-09 \backslash \mathrm{RMSF}=1.994 \mathrm{e}-06 \backslash \mathrm{Dipole}=0.0230435,-0.0760553,0 . \backslash \mathrm{PG}$ $=\mathrm{CS} \quad[\mathrm{SG}(\mathrm{C} 3 \mathrm{H} 3), \mathrm{X}(\mathrm{H} 2)] \backslash \backslash €$

\section{UMP 2/6-31G (d)}

$1 \backslash 1 \backslash G I N C-S C 119 \backslash F T S \backslash U M P 2-F C \backslash 6-31 G(d) \backslash C 3 H 5(2) \backslash R G B 501 \backslash 16-J u n-2002 \backslash 0 \backslash \backslash \# N M$ P2 6-31G* SCF=DIRECT OPT=(TIGHT, MAXCYC=99, TS, CALCFC, NOEIGENTEST) FREQ MAXDISK $=6553600000 \backslash \backslash \mathrm{TS} \mathrm{CH} 3+\mathrm{CHCH}$ Rotational potencial opt and Freq MP2\ $\backslash 0,2 \backslash \mathrm{C},-0.3442438562,0 .,-1.4390914466 \backslash \mathrm{C},-0.3227913195,0 ., 0.7056781943 \backslash$ $\mathrm{C}, 0.7736097843,0 ., 1.2020284857 \backslash \mathrm{H}, 1.809059185,0 ., 1.4512792454 \backslash \mathrm{H},-1.3918$ $38991,0 ., 0.7305714225 \backslash \mathrm{H}, 0.7048144466,0 .,-1.7064267018 \backslash \mathrm{H},-0.8807411462$, $0.9200082779,-1.6435576833 \backslash \mathrm{H},-0.8807411462,-0.9200082779,-1.6435576833$ $\backslash \backslash$ Version=DEC-AXP-OSF /1-G98RevA. 11.3 State=2-A ' \HF=-116.3537811 \MP $2=-1$ $16.7024596 \backslash \mathrm{PUHF}=-116.3753336 \backslash \mathrm{PMP} 2-0=-116.7210002 \backslash \mathrm{S} 2=1.003674 \backslash \mathrm{S} 2-1=0.92$ $6261 \backslash \mathrm{S} 2 \mathrm{~A}=0.789507 \backslash \mathrm{RMSD}=9.444 \mathrm{e}-09 \backslash \mathrm{RMSF}=9.114 \mathrm{e}-08 \backslash \mathrm{Dipole}=0.000108,0 .,-0$. $0919987 \backslash \mathrm{PG}=\mathrm{CS} \quad[\mathrm{SG}(\mathrm{C} 3 \mathrm{H} 3), \mathrm{X}(\mathrm{H} 2)] \backslash \backslash @$

\section{UQCISD / 6-31G (d)}

$1 \backslash 1 \backslash G I N C-S C 87 \backslash F T S \backslash U Q C I S D-F C \backslash 6-31 G(d) \backslash C 3 H 5(2) \backslash R G B 501 \backslash 19-S e p-2002 \backslash 1 \backslash \backslash \# N$ 
QCISD 6-31G* FOPT=(TS, Z-MATRIX, TIGHT, NOEIGENTEST) FREQ MAXDISK=6553600

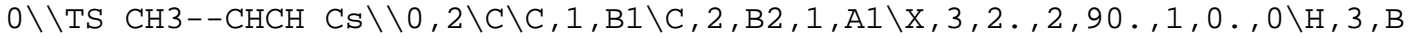
$4,2, \mathrm{~A} 2,4,0 ., 0 \backslash \mathrm{H}, 2, \mathrm{~B} 5,1, \mathrm{~A} 3,3,180.0 \backslash \mathrm{H}, 1, \mathrm{~B} 6,2, \mathrm{~A} 4,3,0 ., 0 \backslash \mathrm{H}, 1, \mathrm{~B} 7,2, \mathrm{~A} 5,7, \mathrm{D} 5$ , $0 \backslash \mathrm{H}, 1, \mathrm{~B} 7,2, \mathrm{~A} 5,7,-\mathrm{D} 5,0 \backslash \backslash \mathrm{B} 1=2.24759724 \backslash \mathrm{B} 2=1.23660414 \backslash \mathrm{B} 4=1.0704955 \backslash \mathrm{B} 5=1$. $07292993 \backslash B 6=1.08595305 \backslash B 7=1.0873167 \backslash A 1=116.50842196 \backslash A 2=161.7129177 \backslash A 3=$

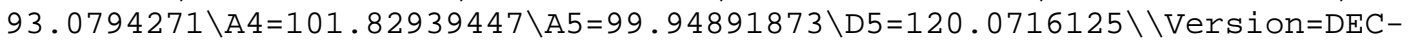
AXP-OSF / 1-G98RevA.11.3 St ate $=2-A^{\prime} \backslash \mathrm{HF}=-116.3564605 \backslash \mathrm{MP} 2=-116.6985208 \backslash \mathrm{MP} 3$ $=-116.730002 \backslash \mathrm{MP} 4 \mathrm{D}=-116.7403891 \backslash \mathrm{MP} 4 \mathrm{DQ}=-116.7343831 \backslash \mathrm{PUHF}=-116.3841808 \backslash \mathrm{PM}$ $\mathrm{P} 2-0=-116.7230956 \backslash \mathrm{PMP} 3-0=-116.7495078 \backslash \mathrm{MP} 4 \mathrm{SDQ}=-116.7416901 \backslash \mathrm{QCISD}=-116.7$ $564859 \backslash \mathrm{S} 2=1.124206 \backslash \mathrm{S} 2-1=1.026964 \backslash \mathrm{S} 2 \mathrm{~A}=0.8416 \backslash \mathrm{RMSD}=5.218 \mathrm{e}-09 \backslash \mathrm{RMSF}=1.165 \mathrm{e}$ $-06 \backslash \mathrm{Dipole}=0.0050009,0 .,-0.117744 \backslash \mathrm{PG}=\mathrm{CS} \quad[\mathrm{SG}(\mathrm{C} 3 \mathrm{H} 3), \mathrm{X}(\mathrm{H} 2)] \backslash \backslash \mathrm{C}$

\section{$\operatorname{UCCSD}(\mathrm{T}) / 6-311 \mathrm{G}(\mathrm{d}, \mathrm{p})$}

$1 \backslash 1 \backslash G I N C-S C 54 \backslash F T S \backslash U C C S D(T)-F C \backslash 6-311 G(d, p) \backslash C 3 H 5(2) \backslash R G B 501 \backslash 18-S e p-2002 \backslash 1$ $\backslash \backslash \# \operatorname{CCSD}(\mathrm{T}) / 6-311 \mathrm{G} * \star \mathrm{SCF}=\mathrm{DIRECT}$ OPT=(TS, EF, Z-MATRIX) FREQ MAXDISK=1048 $57600 \backslash \backslash r e o p t i m i z a c i o n$ y Freqs $\operatorname{CCSD}(\mathrm{T}) / 6-311 \mathrm{G} *$ on Aces geom $\backslash \backslash 0,2 \backslash \mathrm{C} \backslash \mathrm{C}, 1$ , $\mathrm{B} 1 \backslash \mathrm{C}, 2, \mathrm{~B} 2,1, \mathrm{~A} 1 \backslash \mathrm{X}, 3,2 ., 2,90 ., 1,0 ., 0 \backslash \mathrm{H}, 3, \mathrm{~B} 4,2, \mathrm{~A} 2,4,0 ., 0 \backslash \mathrm{H}, 2, \mathrm{~B} 5,1, \mathrm{~A} 3,3,1$ $80 ., 0 \backslash \mathrm{H}, 1, \mathrm{~B} 6,2, \mathrm{~A} 4,3,0 ., 0 \backslash \mathrm{H}, 1, \mathrm{~B} 7,2, \mathrm{~A} 5,7, \mathrm{D} 5,0 \backslash \mathrm{H}, 1, \mathrm{~B} 7,2, \mathrm{~A} 5,7,-\mathrm{D} 5,0 \backslash \backslash \mathrm{B} 1=2$. $24145047 \backslash B 2=1.23520235 \backslash B 4=1.06925295 \backslash B 5=1.0720352 \backslash B 6=1.08654496 \backslash B 7=1.0$ $8806727 \backslash A 1=115.2076507 \backslash A 2=164.3898064 \backslash A 3=92.27196431 \backslash A 4=101.94657039 \backslash A$ $5=99.5538287 \backslash \mathrm{D} 5=120.0299026 \backslash \backslash$ Version=DEC-AXP-OSF $/ 1-G 98$ RevA.11.3 State $=$ $2-A^{\prime} \backslash H F=-116.3900894 \backslash M P 2=-116.7826981 \backslash M P 3=-116.8163935 \backslash M P 4 D=-116.82777$ $12 \backslash \mathrm{MP} 4 \mathrm{DQ}=-116.8195052 \backslash \mathrm{PUHF}=-116.4155793 \backslash \mathrm{PMP} 2-0=-116.8051534 \backslash \mathrm{PMP} 3-0=-11$ $6.8339212 \backslash \mathrm{MP} 4 \mathrm{SDQ}=-116.8267838 \backslash \mathrm{CCSD}=-116.8377641 \backslash \mathrm{CCSD}(\mathrm{T})=-116.8559454 \backslash \mathrm{S}$ $2=1.087882 \backslash S 2-1=0.997087 \backslash S 2 A=0.823505 \backslash R M S D=9.626 e-09 \backslash R M S F=1.626 e-04 \backslash P G$ $=\mathrm{CS} \quad[\mathrm{SG}(\mathrm{C} 3 \mathrm{H} 3), \mathrm{X}(\mathrm{H} 2)] \backslash \backslash \mathrm{Q}$

\section{$\mathrm{CH}_{3}-\mathrm{CH}=\mathrm{CH} \cdot$ (trans)}

\section{$\mathrm{UHF} / 6-31 \mathrm{G}(\mathrm{d})$}

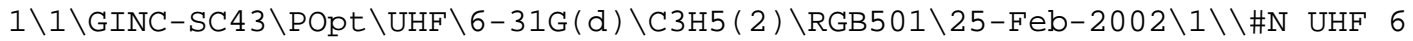
$-31 \mathrm{G} * \mathrm{SCF}=\mathrm{DIRECT}$ OPT=Z-MATRIX FREQ MAXDISK=13107200 \\methyl+acetylene

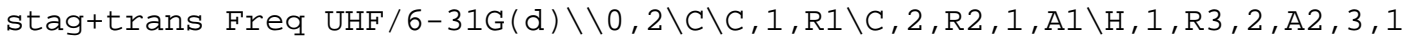
$80 ., 0 \backslash \mathrm{H}, 2, \mathrm{R} 4,1, \mathrm{~A} 3,3,180 ., 0 \backslash \mathrm{H}, 3, \mathrm{R} 5,2, \mathrm{~A} 4,1,0.0 \backslash \mathrm{X}, 3,1 ., 2, \mathrm{~A} 5,6,180 ., 0 \backslash \mathrm{H}, 3$ $, \mathrm{R} 6,7, \mathrm{~A} 6,2, \mathrm{~d}, 0 \backslash \mathrm{H}, 3, \mathrm{R} 6,7, \mathrm{~A} 6,2,-\mathrm{d}, 0 \backslash \backslash \mathrm{R} 1=1.32860747 \backslash \mathrm{R} 2=1.50577856 \backslash \mathrm{R} 3=1.07$ $138748 \backslash R 4=1.08287543 \backslash R 5=1.08319722 \backslash R 6=1.08665165 \backslash A 1=124.74825279 \backslash A 2=13$ $4.03473409 \backslash A 3=118.75484477 \backslash A 4=111.2781558 \backslash A 5=78.79653837 \backslash A 6=66.6164731$ $2 \backslash d=118.71575077 \backslash \backslash$ Version=DEC-AXP-OSF $/ 1-G 98$ RevA. 9 $\backslash$ State $=2-A^{\prime} \backslash H F=-116.4$ $29146 \backslash S 2=1.000685 \backslash S 2-1=0 . \backslash S 2 A=0.757771 \backslash R M S D=4.672 e-09 \backslash R M S F=5.561 e-05 \backslash D$ ipole $=-0.1590083,0 ., 0.1755482 \backslash \mathrm{PG}=\mathrm{CS} \quad[\mathrm{SG}(\mathrm{C} 3 \mathrm{H} 3), \mathrm{X}(\mathrm{H} 2)] \backslash \backslash @$

\section{UB3LYP / 6-31G (d)}

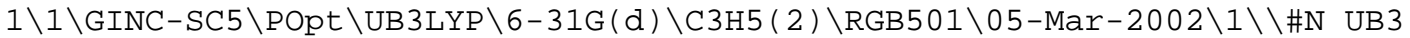
LYP 6-31G* SCF=DIRECT OPT=Z-MATRIX FREQ MAXDISK=13107200 \methyl+acety

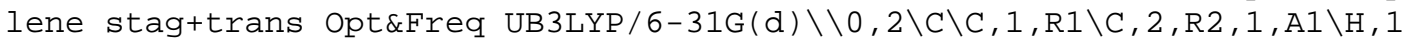
, R3, 2, A2, 3, $180 ., 0 \backslash \mathrm{H}, 2, \mathrm{R} 4,1, \mathrm{~A} 3,3,180 ., 0 \backslash \mathrm{H}, 3, \mathrm{R} 5,2, \mathrm{~A} 4,1,0 ., 0 \backslash \mathrm{X}, 3,1 ., 2, \mathrm{~A} 5$, $6,180 ., 0 \backslash H, 3, R 6,7, A 6,2, d, 0 \backslash H, 3, R 6,7, A 6,2,-d, 0 \backslash \backslash R 1=1.31354279 \backslash R 2=1.5089$ $3965 \backslash R 3=1.08302098 \backslash R 4=1.09927592 \backslash R 5=1.09339158 \backslash R 6=1.09769835 \backslash A 1=125.91$ $076668 \backslash \mathrm{A} 2=136.99124345 \backslash \mathrm{A} 3=118.52279906 \backslash \mathrm{A} 4=111.2035635 \backslash \mathrm{A} 5=78.34693903 \backslash \mathrm{A}$ $6=66.71813087 \backslash \mathrm{d}=118.9293312 \backslash \backslash$ Version=DEC-AXP-OSF /1-G98RevA.9 $\backslash$ State $=2-A$ ' $\backslash \mathrm{HF}=-117.2203836 \backslash \mathrm{S} 2=0.76094 \backslash \mathrm{S} 2-1=0 . \backslash \mathrm{S} 2 \mathrm{~A}=0.750042 \backslash \mathrm{RMSD}=4.185 e-09 \backslash \mathrm{RMSF}=$ $3.629 e-05 \backslash \mathrm{Dipole}=-0.1611701,0 ., 0.216935 \backslash \mathrm{PG}=\mathrm{CS} \quad[\mathrm{SG}(\mathrm{C} 3 \mathrm{H} 3), \mathrm{X}(\mathrm{H} 2)] \backslash \backslash \mathrm{Q}$

\section{UB3LYP / 6-31G (2df, p)}

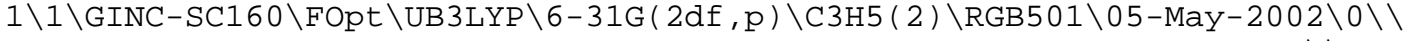
\#N UB3LYP 6-31G (2DF,P) TEST SCF=DIRECT OPT FREQ MAXDISK=117964800 \met hyl+acetylene stag+trans Opt\&Freq UB3LYP/6-31G (2df,p) \\0,2\C, -0.449570 $1615,0 \ldots,-1.2749008401 \backslash \mathrm{C},-0.4429553976,0 ., 0.0335908391 \backslash \mathrm{C}, 0.7780787265,0$ $.0 .9162800972 \backslash \mathrm{H},-1.1751955016,0 .,-2.0746698941 \backslash \mathrm{H},-1.4051636054,0 ., 0.5$ 
$622537977 \backslash \mathrm{H}, 1.695387944,0.0 .3239164677 \backslash \mathrm{H}, 0.7858260793,0.8806819245,1$. $5693395258 \backslash \mathrm{H}, 0.7858260793,-0.8806819245,1.5693395258 \backslash \backslash$ Version=DEC $-\mathrm{AXP}-$ $\mathrm{OSF} / 1-\mathrm{G} 98 \mathrm{RevA} .11 .3 \backslash$ State $=2-\mathrm{A}^{\prime} \backslash \mathrm{HF}=-117.2309571 \backslash \mathrm{S} 2=0.759946 \backslash \mathrm{S} 2-1=0 . \backslash \mathrm{S} 2 \mathrm{~A}=$ $0.750042 \backslash \mathrm{RMSD}=5.819 \mathrm{e}-09 \backslash \mathrm{RMSF}=2.373 e-05 \backslash \mathrm{Dipole}=-0.1456737,0 ., 0.2201095 \backslash$ $\mathrm{PG}=\mathrm{CS} \quad[\mathrm{SG}(\mathrm{C} 3 \mathrm{H} 3), \mathrm{X}(\mathrm{H} 2)] \backslash \backslash \mathrm{Q}$

\section{UB3LYP/CC-PVTZ}

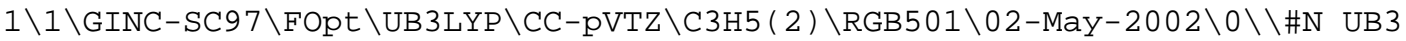
LYP CC-PVTZ TEST SCF=DIRECT OPT FREQ MAXDISK=117964800\\methyl+acetyle

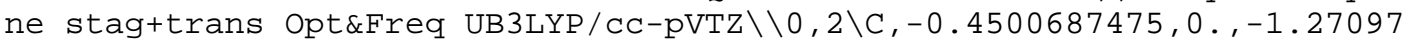
$97769 \backslash \mathrm{C},-0.4410132185,0 ., 0.033191868 \backslash \mathrm{C}, 0.7777801898,0 ., 0.9145368058 \backslash \mathrm{H}$, $-1.1746431255,0 .,-2.0683749034 \backslash \mathrm{H},-1.4032202665,0 ., 0.5556704081 \backslash \mathrm{H}, 1.691$ $730035,0.0 .3239070424 \backslash \mathrm{H}, 0.782972007,0.8782733172,1.5641520358 \backslash \mathrm{H}, 0.782$ $972007,-0.8782733172,1.5641520358 \backslash \backslash$ Version=DEC-AXP-OSF / 1-G98RevA.11.3\ State $=2-A^{\prime} \backslash H F=-117.2691867 \backslash S 2=0.758988 \backslash S 2-1=0 . \backslash S 2 A=0.750039 \backslash R M S D=6.547$ $e-09 \backslash \mathrm{RMSF}=2.211 \mathrm{e}-05 \backslash \mathrm{Dipole}=-0.154836,0 ., 0.2515735 \backslash \mathrm{PG}=\mathrm{CS} \quad[\mathrm{SG}(\mathrm{C} 3 \mathrm{H} 3), \mathrm{X}(\mathrm{H} 2$ )$] \backslash \backslash 0$

\section{UB3LYP / 6-311+G (3df, 2p)}

$1 \backslash 1 \backslash G I N C-S C 83 \backslash F O p t \backslash U B 3 L Y P \backslash 6-311+G(3 d f, 2 p) \backslash C 3 H 5$ (2) $\backslash R G B 501 \backslash 15-M a r-2003 \backslash 0$ $\backslash \backslash \# \mathrm{~N}$ UB3LYP $6-311+\mathrm{G}(3 \mathrm{DF}, 2 \mathrm{P}) \quad \mathrm{SCF}=\mathrm{TIGHT}$ TEST OPT=TIGHT FREQ MAXDISK=3932

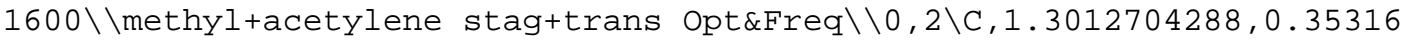
$92801,0 . \backslash \mathrm{C}, 0.000013099,0.4424435382,0 . \backslash \mathrm{C},-0.9707727782,-0.7065855054,0$ $. \backslash \mathrm{H}, 2.1538844795,1.011857477,0 . \backslash \mathrm{H},-0.4478544406,1.4418091677,0 . \backslash \mathrm{H},-0.4$ $518019631,-1.6629763556,0 . \backslash \mathrm{H},-1.6186462865,-0.6624270833,0.8783040507 \backslash$ $\mathrm{H},-1.6186462865,-0.6624270833,-0.8783040507 \backslash \backslash$ Version=DEC-AXP $-O S F / 1-G 98$ RevA.11.3 State=2-A ' $\backslash H F=-117.2663521 \backslash S 2=0.758921 \backslash S 2-1=0 . \backslash S 2 A=0.750039 \backslash$ $\mathrm{RMSD}=5.544 \mathrm{e}-09 \backslash \mathrm{RMSF}=2.833 \mathrm{e}-06 \backslash \mathrm{Dipole}=-0.257222,0.1728279,0 . \backslash \mathrm{PG}=\mathrm{CS} \quad[S G($ $\mathrm{C} 3 \mathrm{H} 3), \mathrm{X}(\mathrm{H} 2)] \backslash \backslash @$

\section{UMPW1K/6-31+G (d, p)}

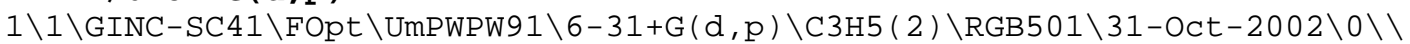
\#MPWPW91 6-31+G** IOP (5/45=10000428) IOP $(5 / 46=05720572) \quad$ IOP $(5 / 47=10001$

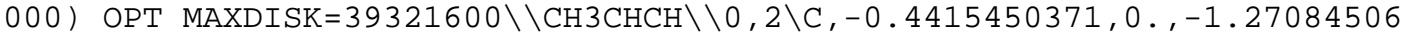
$37 \backslash \mathrm{C},-0.4413158566,0.0 .0377817809 \backslash \mathrm{C}, 0.7723031236,0.0 .9115828435 \backslash \mathrm{H},-1$ $.1748378848,0 .,-2.0575376145 \backslash \mathrm{H},-1.4035893132,0 ., 0.5507724879 \backslash \mathrm{H}, 1.68263$ $81452,0.0 .3196255484 \backslash \mathrm{H}, 0.7795678366,0.8768730733,1.5580111069 \backslash \mathrm{H}, 0.779$ $5678366,-0.8768730733,1.5580111069 \backslash \backslash$ Version=DEC-AXP-OSF /1-G98RevA.11.3 $\backslash$ State $=2-A^{\prime} \backslash \mathrm{HF}=-117.1979076 \backslash \mathrm{S} 2=0.773624 \backslash \mathrm{S} 2-1=0 . \backslash \mathrm{S} 2 \mathrm{~A}=0.750162 \backslash \mathrm{RMSD}=9.94$ $2 e-09 \backslash \mathrm{RMSF}=1.245 e-04 \backslash \mathrm{Dipole}=-0.1783904,0 ., 0.2577511 \backslash \mathrm{PG}=\mathrm{CS} \quad[\mathrm{SG}(\mathrm{C} 3 \mathrm{H} 3), \mathrm{X}($ $\mathrm{H} 2)] \backslash \backslash @$

\section{UMP 2/6-31G (d)}

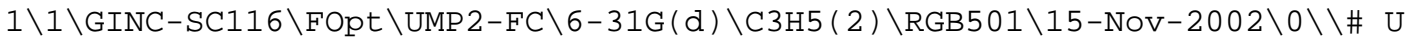

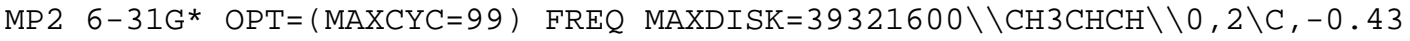
$61429619,0 \ldots,-1.2619770469 \backslash \mathrm{C},-0.4444603,0.0 .028723438 \backslash \mathrm{C}, 0.7720758729,0$ $., 0.9119864307 \backslash \mathrm{H},-1.1775332785,0 .,-2.0481665539 \backslash \mathrm{H},-1.4108747886,0 ., 0.5$ $435984416 \backslash \mathrm{H}, 1.684166425,0.0 .3132843016 \backslash \mathrm{H}, 0.7777029881,0.8821072956,1$. $5594434399 \backslash \mathrm{H}, 0.7777029881,-0.8821072956,1.5594434399 \backslash \backslash$ Version=DEC $-\mathrm{AXP}-$ OSF /1-G98RevA.11.3 \State $=2-A^{\prime} \backslash \mathrm{HF}=-116.4273503 \backslash \mathrm{MP} 2=-116.7738105 \backslash \mathrm{PUHF}=-1$ $16.4382661 \backslash \mathrm{PMP} 2-0=-116.7834849 \backslash \mathrm{S} 2=0.927276 \backslash \mathrm{S} 2-1=0.886307 \backslash \mathrm{S} 2 \mathrm{~A}=0.754803 \backslash$ $\mathrm{RMSD}=7.022 \mathrm{e}-09 \backslash \mathrm{RMSF}=1.473 \mathrm{e}-04 \backslash \mathrm{Dipole}=-0.1788542,0 ., 0.1977055 \backslash \mathrm{PG}=\mathrm{CS}$ [SG ( $\mathrm{C} 3 \mathrm{H} 3), \mathrm{X}(\mathrm{H} 2)] \backslash \backslash @$

\section{UQCISD/6-31G (d)}

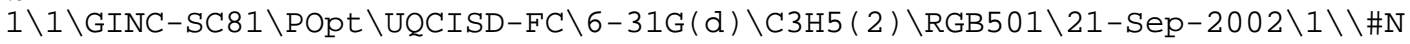
UQCISD 6-31G* SCF $=(D I R E C T, M A X C Y C=99) \quad O P T=(Z-M A T R I X, M A X C Y C=99) \quad F R E Q$ MA

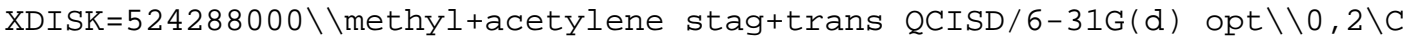
$\backslash \mathrm{C}, 1, \mathrm{R} 1 \backslash \mathrm{C}, 2, \mathrm{R} 2,1, \mathrm{~A} 1 \backslash \mathrm{H}, 1, \mathrm{R} 3,2, \mathrm{~A} 2,3,180 ., 0 \backslash \mathrm{H}, 2, \mathrm{R} 4,1, \mathrm{~A} 3,3,180 ., 0 \backslash \mathrm{H}, 3, \mathrm{R} 5,2$ 


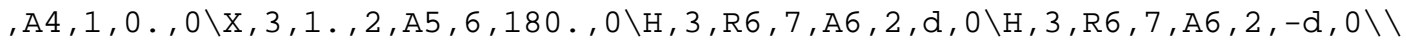
$R 1=1.32134599 \backslash R 2=1.50904298 \backslash R 3=1.08433442 \backslash R 4=1.09849068 \backslash R 5=1.09497566 \backslash$ $R 6=1.09786509 \backslash A 1=125.22316113 \backslash A 2=135.637376 \backslash A 3=118.67308895 \backslash A 4=110.809$ $19826 \backslash A 5=83.2183516 \backslash A 6=64.60910684 \backslash d=116.82538296 \backslash \backslash$ Version=DEC $-A X P-O S F$ /1-G98RevA.11.3 State $=2-A^{\prime} \backslash \mathrm{HF}=-116.4284928 \backslash \mathrm{MP} 2=-116.7724361 \backslash \mathrm{MP} 3=-116.8$ $041211 \backslash \mathrm{MP} 4 \mathrm{D}=-116.8137263 \backslash \mathrm{MP} 4 \mathrm{DQ}=-116.80784 \backslash \mathrm{PUHF}=-116.4417238 \backslash \mathrm{PMP} 2-0=-11$ $6.7843088 \backslash \mathrm{PMP} 3-0=-116.8136843 \backslash \mathrm{MP} 4 \mathrm{SDQ}=-116.8125916 \backslash \mathrm{QCISD}=-116.8219976 \backslash \mathrm{S}$ $2=0.98673 \backslash \mathrm{S} 2-1=0.937206 \backslash \mathrm{S} 2 \mathrm{~A}=0.757513 \backslash \mathrm{RMSD}=4.525 \mathrm{e}-09 \backslash \mathrm{RMSF}=1.226 \mathrm{e}-05 \backslash \mathrm{Dip}$ ole $=-0.1721816,0 ., 0.1959639 \backslash \mathrm{PG}=\mathrm{CS} \quad[\mathrm{SG}(\mathrm{C} 3 \mathrm{H} 3), \mathrm{X}(\mathrm{H} 2)] \backslash \backslash @$

\section{$\operatorname{UCCSD}(\mathrm{T}) / 6-311 \mathrm{G}(\mathrm{d}, \mathrm{p})$}

$1 \backslash 1 \backslash G I N C-S C 117 \backslash P O p t \backslash U C C S D(T)-F C \backslash 6-311 G(d, p) \backslash C 3 H 5(2) \backslash R G B 501 \backslash 25-O c t-2002$ $\backslash 1 \backslash \backslash \# \mathrm{~N}$ UCCSD $(\mathrm{T})$ 6-311G** $\mathrm{SCF}=(\mathrm{DIRECT}, \mathrm{MAXCYC}=99, \mathrm{RESTART})$ OPT=(Z-MATRIX, MAXCYC=99) MAXDISK=262144000 FREQ \\methyl+acetylene stag+trans opt \& $f$ req $\backslash \backslash 0,2 \backslash C \backslash C, 1, R 1 \backslash C, 2, R 2,1, A 1 \backslash H, 1, R 3,2, A 2,3,180 ., 0 \backslash H, 2, R 4,1, A 3,3,180$. , $0 \backslash \mathrm{H}, 3, \mathrm{R} 5,2, \mathrm{~A} 4,1,0 ., 0 \backslash \mathrm{X}, 3,1 ., 2, \mathrm{~A} 5,6,180 ., 0 \backslash \mathrm{H}, 3, \mathrm{R} 6,7, \mathrm{~A} 6,2, \mathrm{~d}, 0 \backslash \mathrm{H}, 3, \mathrm{R} 6,7, \mathrm{~A}$ $6,2,-d, 0 \backslash \backslash R 1=1.32391749 \backslash R 2=1.51413486 \backslash R 3=1.08431691 \backslash R 4=1.09938752 \backslash R 5=1$ $.09489428 \backslash R 6=1.09813712 \backslash A 1=125.13364602 \backslash A 2=136.07134585 \backslash A 3=118.5253332$ $8 \backslash A 4=110.72679487 \backslash A 5=81.52077398 \backslash A 6=65.27275053 \backslash d=117.25848588 \backslash \backslash$ Versio $\mathrm{n}=\mathrm{DEC}-\mathrm{AXP}-\mathrm{OSF} / 1-\mathrm{G} 98 \mathrm{RevA} .11 .3 \backslash \mathrm{State}=2-\mathrm{A}^{\prime} \backslash \mathrm{HF}=-116.4589345 \backslash \mathrm{MP} 2=-116.85209$ $17 \backslash \mathrm{MP} 3=-116.8867574 \backslash \mathrm{MP} 4 \mathrm{D}=-116.8973484 \backslash \mathrm{MP} 4 \mathrm{DQ}=-116.8892739 \backslash \mathrm{PUHF}=-116.471$ $7634 \backslash \mathrm{PMP} 2-0=-116.8635767 \backslash \mathrm{PMP} 3-0=-116.8959409 \backslash \mathrm{MP} 4 \mathrm{SDQ}=-116.8940005 \backslash \mathrm{CCSD}=$ $-116.9013416 \backslash \mathrm{CCSD}(\mathrm{T})=-116.917615 \backslash \mathrm{S} 2=0.975827 \backslash \mathrm{S} 2-1=0.927603 \backslash \mathrm{S} 2 \mathrm{~A}=0.75729$ $8 \backslash \mathrm{RMSD}=5.534 \mathrm{e}-09 \backslash \mathrm{RMSF}=8.132 \mathrm{e}-05 \backslash \mathrm{PG}=\mathrm{CS} \quad[\mathrm{SG}(\mathrm{C} 3 \mathrm{H} 3), \mathrm{X}(\mathrm{H} 2)] \backslash \backslash \mathrm{Q}$

\section{$\mathrm{CH}_{2}=\mathrm{CH}-\mathrm{CH}_{3}$}

\section{RQCISD/6-31G (d)}

$1 \backslash 1 \backslash G I N C-S C 51 \backslash F O p t \backslash R Q C I S D-F C \backslash 6-31 G(d) \backslash C 3 H 6 \backslash R G B 501 \backslash 23-O c t-2002 \backslash 0 \backslash \backslash \# Q C I$

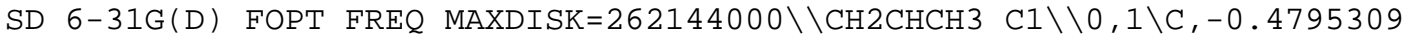
$422,0.1733289839,-1.1985921938 \backslash \mathrm{C},-0.4435829243,-0.1541705131,0.0992837$ $148 \backslash \mathrm{C}, 0.7698166746,-0.0325526473,0.9802854788 \backslash \mathrm{H}, 0.3976550136,0.5619991$ $855,-1.7151790638 \backslash \mathrm{H}, 1.6262010768,0.3606530229,0.4195266921 \backslash \mathrm{H}, 0.5769646$ $653,0.63884874,1.8280582462 \backslash \mathrm{H}, 1.052667013,-1.0079970742,1.3990322127 \backslash \mathrm{H}$ $,-1.3484807676,-0.5395002196,0.574498279 \backslash \mathrm{H},-1.3852238502,0.0663614044$, $-1.7917983652 \backslash \backslash$ Version=DEC-AXP-OSF /1-G98RevA.11.3 $\backslash \mathrm{HF}=-117.0702763 \backslash \mathrm{MP} 2=$ $-117.4554848 \backslash \mathrm{MP} 3=-117.485627 \backslash \mathrm{MP} 4 \mathrm{D}=-117.4963472 \backslash \mathrm{MP} 4 \mathrm{DQ}=-117.4892027 \backslash \mathrm{MP} 4 \mathrm{~S}$ $\mathrm{DQ}=-117.4929151 \backslash \mathrm{QCISD}=-117.4951527 \backslash \mathrm{RMSD}=2.895 \mathrm{e}-09 \backslash \mathrm{RMSF}=3.241 \mathrm{e}-05 \backslash \mathrm{Dipol}$ $\mathrm{e}=0.0328149,-0.0168568,0.102161 \backslash \mathrm{PG}=\mathrm{CO} 1 \quad[\mathrm{X}(\mathrm{C} 3 \mathrm{H} 6)] \backslash \backslash @$

\section{$\cdot \mathrm{CH}_{3}---\mathrm{CH}_{2}=\mathrm{CH}-\mathrm{CH}_{3}$}

\section{UQCISD/6-31G (d)}

$1 \backslash 1 \backslash G I N C-S C 35 \backslash F T S \backslash U Q C I S D-F C \backslash 6-31 G(d) \backslash C 4 H 9(2) \backslash R G B 501 \backslash 01-O c t-2002 \backslash 1 \backslash \backslash \# N$ QCISD 6-31G* FOPT=(TS, Z-MATRIX, TIGHT, NOEIGENTEST) FREQ MAXDISK=1310720 $00 \backslash \backslash \mathrm{TS} 3$ geom opt for $\mathrm{W} 1 \backslash \backslash 0,2 \backslash \mathrm{C} \backslash \mathrm{C}, 1, \mathrm{~B} 1 \backslash \mathrm{C}, 2, \mathrm{~B} 2,1, \mathrm{~A} 1 \backslash \mathrm{C}, 1, \mathrm{~B} 3,2, \mathrm{~A} 2,3, \mathrm{D} 1,0 \backslash \mathrm{H}$ , 4, B4, 1, A3, 2, D2, $0 \backslash \mathrm{H}, 3, \mathrm{~B} 5,2, \mathrm{~A} 4,1, \mathrm{D} 3,0 \backslash \mathrm{H}, 3, \mathrm{~B} 6,2, \mathrm{~A} 5,6, \mathrm{D} 4,0 \backslash \mathrm{H}, 3, \mathrm{~B} 7,2, \mathrm{~A} 6,6$, $\mathrm{D} 5,0 \backslash \mathrm{H}, 2, \mathrm{~B} 8,1, \mathrm{~A} 7,3, \mathrm{D} 6,0 \backslash \mathrm{H}, 1, \mathrm{~B} 9,2, \mathrm{~A} 8,4, \mathrm{D} 7,0 \backslash \mathrm{H}, 1, \mathrm{~B} 10,2, \mathrm{~A} 9,4, \mathrm{D} 8,0 \backslash \mathrm{H}, 4, \mathrm{~B} 11$ , 1, A10, 5, D9, $0 \backslash \mathrm{H}, 4, \mathrm{~B} 12,1, \mathrm{~A} 11,5, \mathrm{D} 10,0 \backslash \backslash \mathrm{B} 1=1.36722267 \backslash \mathrm{B} 2=1.50196257 \backslash \mathrm{B} 3=2$. $27569535 \backslash B 4=1.08708351 \backslash B 5=1.09888278 \backslash B 6=1.09718233 \backslash B 7=1.10034625 \backslash B 8=1$. $09155073 \backslash \mathrm{B} 9=1.08893693 \backslash \mathrm{B} 10=1.08748045 \backslash \mathrm{B} 11=1.08817095 \backslash \mathrm{B} 12=1.08686995 \backslash \mathrm{A} 1$ $=123.9386049 \backslash A 2=109.21074892 \backslash A 3=101.35679526 \backslash A 4=111.33704528 \backslash A 5=111.01$ $640458 \backslash A 6=111.41882205 \backslash A 7=118.94000728 \backslash A 8=120.03812972 \backslash A 9=120.26501384$ $\backslash A 10=100.52211419 \backslash A 11=101.66797054 \backslash D 1=84.86050977 \backslash D 2=-59.936699 \backslash D 3=130$ $.24727376 \backslash D 4=-120.45345055 \backslash D 5=119.27313215 \backslash D 6=-172.97130124 \backslash D 7=-100.89$ $329294 \backslash D 8=102.46603554 \backslash D 9=-119.89915464 \backslash D 10=120.0856708 \backslash \backslash$ Version=DEC $-A$ $\mathrm{XP}-\mathrm{OSF} / 1-\mathrm{G} 98 \mathrm{RevA} .11 .3 \backslash \mathrm{HF}=-156.613469 \backslash \mathrm{MP} 2=-157.1009825 \backslash \mathrm{MP} 3=-157.1484655$ $\backslash \mathrm{MP} 4 \mathrm{D}=-157.1622782 \backslash \mathrm{MP} 4 \mathrm{DQ}=-157.1544544 \backslash \mathrm{PUHF}=-156.6323375 \backslash \mathrm{PMP} 2-0=-157.11$ 
$73458 \backslash \mathrm{PMP} 3-0=-157.1611547 \backslash \mathrm{MP} 4 \mathrm{SDQ}=-157.1605522 \backslash \mathrm{QCISD}=-157.1704648 \backslash \mathrm{S} 2=1$. $013165 \backslash S 2-1=0.941603 \backslash \mathrm{S} 2 \mathrm{~A}=0.760661 \backslash \mathrm{RMSD}=7.666 \mathrm{e}-09 \backslash \mathrm{RMSF}=1.698 \mathrm{e}-07 \backslash \mathrm{Dipole}$ $=0.0244786,-0.0091142,0.0329946 \backslash \mathrm{PG}=\mathrm{C} 01 \quad[\mathrm{X}(\mathrm{C} 4 \mathrm{H} 9)] \backslash \backslash @$

\section{$\mathrm{CH}_{3}-\mathrm{CH}_{2}-\mathrm{CH}\left(\mathrm{CH}_{3}\right) \cdot$}

\section{UQCISD/6-31G (d)}

$1 \backslash 1 \backslash G I N C-S C 121 \backslash F O p t \backslash U Q C I S D-F C \backslash 6-31 G(d) \backslash C 4 H 9$ (2) $\backslash$ RGB $501 \backslash 28-N o v-2002 \backslash 0 \backslash \backslash \#$ $\mathrm{N} \# \mathrm{~N}$ QCISD 6-31G* OPT FREQ MAXDISK=262144000 \CH3CH2CHCH3 Rotado $\backslash 0,2 \backslash$ $\mathrm{C}, 0.184022431,-0.7471285009,0.046599334 \backslash \mathrm{C},-0.039626874,0.7329656468,-0$ $.0146846357 \backslash \mathrm{C},-0.6221589979,-1.4367777897,1.1508759892 \backslash \mathrm{C}, 0.4464917499$, $1.5337548009,-1.1815900741 \backslash \mathrm{H},-0.0653767959,-1.1925140702,-0.9301586351$ $\backslash \mathrm{H}, 1.2600564282,-0.9619510315,0.1956093763 \backslash \mathrm{H},-0.4273880588,-2.51623450$ $78,1.1708774868 \backslash \mathrm{H},-0.3621179924,-1.0294508885,2.136554661 \backslash \mathrm{H},-1.6980868$ $652,-1.2867064839,0.9984132478 \backslash \mathrm{H},-0.2676361047,1.2546318997,0.91588547$ $96 \backslash \mathrm{H}, 0.0217432207,2.5449474414,-1.1837111725 \backslash \mathrm{H}, 1.5462627586,1.64098387$ $35,-1.1786652558 \backslash \mathrm{H}, 0.1801735552,1.0494088245,-2.1320088691 \backslash \backslash$ Version=DE $\mathrm{C}-\mathrm{AXP}-\mathrm{OSF} / 1-\mathrm{G} 98 \mathrm{RevA} .11 .3 \backslash \mathrm{HF}=-156.6697028 \backslash \mathrm{MP} 2=-157.1702791 \backslash \mathrm{MP} 3=-157.216$ $1218 \backslash \mathrm{MP} 4 \mathrm{D}=-157.2293488 \backslash \mathrm{MP} 4 \mathrm{DQ}=-157.2213629 \backslash \mathrm{PUHF}=-156.6731173 \backslash \mathrm{PMP} 2-0=-15$ $7.1724881 \backslash \mathrm{PMP} 3-0=-157.2174501 \backslash \mathrm{MP} 4 \mathrm{SDQ}=-157.2253036 \backslash \mathrm{QCISD}=-157.2282756 \backslash \mathrm{S}$ $2=0.763533 \backslash \mathrm{S} 2-1=0.753919 \backslash \mathrm{S} 2 \mathrm{~A}=0.750137 \backslash \mathrm{RMSD}=2.920 \mathrm{e}-09 \backslash \mathrm{RMSF}=1.460 \mathrm{e}-06 \backslash \mathrm{D} i$ pole $=-0.0638417,0.0623083,-0.0172878 \backslash \mathrm{PG}=\mathrm{C} 01 \quad[\mathrm{X}(\mathrm{C} 4 \mathrm{H} 9)] \backslash \backslash \mathrm{Q}$

\section{$\mathrm{CH} \equiv \mathrm{C}-\mathrm{CH}_{3}$}

\section{RQCISD/6-31G (d)}

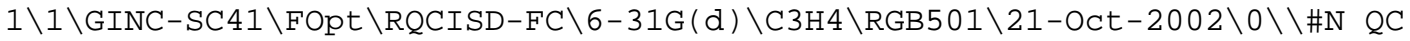
ISD 6-31G* OPT FREQ TEST MAXDISK=39321600\\Propine Opt and Freq $\backslash \backslash 0,1 \backslash \mathrm{C}$ $, 0.0032005422,1.4329423796,0 . \backslash \mathrm{C}, 0.0002388424,0.2195191892,0 . \backslash \mathrm{H}, 0.00557$ $24203,2.5015689937,0 . \backslash \mathrm{C},-0.0026598724,-1.2502077484,0 . \backslash \mathrm{H}, 1.0216540236$, $-1.6403449084,0 . \backslash \mathrm{H},-0.5159517583,-1.6373735039,0.8877304798 \backslash \mathrm{H},-0.51595$ $17583,-1.6373735039,-0.8877304798 \backslash \backslash$ Version=DEC-AXP-OSF /1-G98RevA.11.3\ State $=1-A^{\prime} \backslash H F=-115.8623448 \backslash M P 2=-116.2416943 \backslash M P 3=-116.2597795 \backslash M P 4 D=-116$ $.2706341 \backslash \mathrm{MP} 4 \mathrm{DQ}=-116.2630341 \backslash \mathrm{MP} 4 \mathrm{SDQ}=-116.26784 \backslash \mathrm{QCISD}=-116.2697464 \backslash \mathrm{RMSD}=$ $2.706 e-09 \backslash \mathrm{RMSF}=7.851 \mathrm{e}-05 \backslash \mathrm{Dipole}=-0.0004237,-0.2412694,0 . \backslash \mathrm{PG}=\mathrm{CS} \quad[\mathrm{SG}(\mathrm{C} 3 \mathrm{H}$ 2), $\mathrm{X}(\mathrm{H} 2)] \backslash \backslash \mathrm{Q}$

\section{$\cdot \mathrm{CH}_{3}---\mathrm{CH} \equiv \mathrm{C}-\mathrm{CH}_{3}$}

\section{UQCISD/6-31G (d)}

$1 \backslash 1 \backslash G I N C-S C 80 \backslash F T S \backslash U Q C I S D-F C \backslash 6-31 G(d) \backslash C 4 H 7(2) \backslash R G B 501 \backslash 28-S e p-2002 \backslash 1 \backslash \backslash \# N$ QCISD 6-31G* FOPT=(TS, Z-MATRIX, TIGHT, NOEIGENTEST) FREQ MAXDISK=1310720

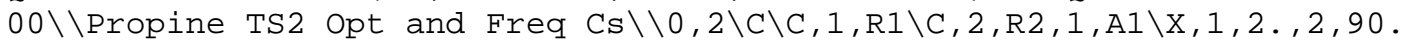

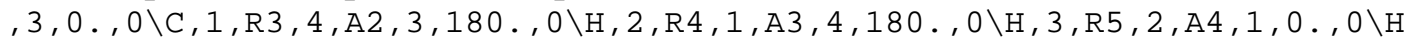
, 3, R6, 2, A5, 6, d1 , $0 \backslash \mathrm{H}, 3, \mathrm{R} 6,2, \mathrm{~A} 5,6,-\mathrm{d} 1,0 \backslash \mathrm{H}, 5, \mathrm{R} 7,1, \mathrm{~A} 6,2,0 ., 0 \backslash \mathrm{H}, 5, \mathrm{R} 8,1, \mathrm{~A} 7,2$ $, \mathrm{d} 2,0 \backslash \mathrm{H}, 5, \mathrm{R} 8,1, \mathrm{~A} 7,2,-\mathrm{d} 2,0 \backslash \backslash \mathrm{R} 1=1.23682551 \backslash \mathrm{R} 2=2.24700682 \backslash \mathrm{R} 3=1.46892901 \backslash \mathrm{R}$ $4=1.07288571 \backslash R 5=1.0865457 \backslash R 6=1.08726326 \backslash R 7=1.09889696 \backslash R 8=1.09659886 \backslash A 1$ $=115.60322973 \backslash A 2=74.1693918 \backslash A 3=150.389847 \backslash A 4=102.00758602 \backslash A 5=100.15681$ $417 \backslash A 6=110.83102061 \backslash A 7=110.89817941 \backslash d 1=59.93927708 \backslash d 2=120.01770277 \backslash \backslash V e$ rsion=DEC-AXP-OSF /1-G98RevA.11.3 State $=2-A^{\prime} \backslash H F=-155.4008857 \backslash M P 2=-155.8$ $758064 \backslash \mathrm{MP} 3=-155.9159213 \backslash \mathrm{MP} 4 \mathrm{D}=-155.929663 \backslash \mathrm{MP} 4 \mathrm{DQ}=-155.9212537 \backslash \mathrm{PUHF}=-155$. $4262211 \backslash \mathrm{PMP} 2-0=-155.8980856 \backslash \mathrm{PMP} 3-0=-155.9332912 \backslash \mathrm{MP} 4 \mathrm{SDQ}=-155.929621 \backslash \mathrm{QCI}$ $\mathrm{SD}=-155.9430467 \backslash \mathrm{S} 2=1.082581 \backslash \mathrm{S} 2-1=0.992437 \backslash \mathrm{S} 2 \mathrm{~A}=0.819523 \backslash \mathrm{RMSD}=4.607 e-09 \backslash$ $\mathrm{RMSF}=1.897 \mathrm{e}-06 \backslash \mathrm{Dipole}=0.0646445,0 .,-0.2247636 \backslash \mathrm{PG}=\mathrm{CS} \quad[\mathrm{SG}(\mathrm{C} 4 \mathrm{H} 3), \mathrm{X}(\mathrm{H} 4)] \backslash \backslash$ C 


\section{$\mathrm{CH}_{3}-\mathrm{CH}=\mathrm{C}\left(\mathrm{CH}_{3}\right) \cdot$ (trans)}

UQCISD/6-31G (d)

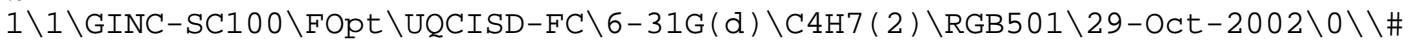
$\mathrm{N}$ QCISD 6-31G* OPT FREQ MAXDISK=131072000\\Propine Opt and Freq HF/6-3 $1 \mathrm{G}(\mathrm{d}) \mathrm{Cs} \backslash \backslash 0,2 \backslash \mathrm{C}, 0.0380564337,0.6313500132,-0.0022981396 \backslash \mathrm{C}, 0.0380564337$ $,-0.6908866368,-0.0022981396 \backslash C,-1.1895873619,-1.5641257097,0.071836416$ $5 \backslash \mathrm{C}, 1.0423770041,1.7199838415,-0.0629467251 \backslash \mathrm{H}, 1.0039843048,-1.21433377$ $87,-0.0606282792 \backslash \mathrm{H},-2.0972655522,-0.9534533077,0.1266489933 \backslash \mathrm{H},-1.15607$ $29292,-2.2144322171,0.9562527344 \backslash \mathrm{H},-1.2627440596,-2.2144322171,-0.8101$ $859971 \backslash \mathrm{H}, 2.060682813,1.3015375509,-0.1244398467 \backslash \mathrm{H}, 0.8855783876,2.35859$ $24603,-0.9413513464 \backslash \mathrm{H}, 0.992421978,2.3585924603,0.8279432683 \backslash \backslash$ Version=D EC-AXP-OSF /1-G98RevA.11.3 State $=2-A \cdot \backslash H F=-155.4716958 \backslash M P 2=-155.9477676 \backslash$ $M P 3=-155.9889813 \backslash M P 4 D=-156.0017235 \backslash M P 4 D Q=-155.9934117 \backslash P U H F=-155.484133$ $6 \backslash P M P 2-0=-155.9588623 \backslash P M P 3-0=-155.997791 \backslash M P 4 S D Q=-155.9993311 \backslash Q C I S D=-15$ $6.0083161 \backslash \mathrm{S} 2=0.96175 \backslash \mathrm{S} 2-1=0.915104 \backslash \mathrm{S} 2 \mathrm{~A}=0.757099 \backslash \mathrm{RMSD}=2.851 \mathrm{e}-09 \backslash \mathrm{RMSF}=2$. $114 e-05 \backslash$ Dipole $=-0.2403424,0 .,-0.0103924 \backslash P G=C S \quad[S G(C 4 H 3), X(H 4)] \backslash \backslash @$ 
TABLE S2: Total Energies (Hartrees) for QCISD/6-31G(d) Geometries, ZPVE Not Included

\begin{tabular}{lcccc}
\hline Level of Theory & $\cdot \mathrm{CH}_{3}$ & $\mathrm{CH}_{2}=\mathrm{CH}_{2}$ & $\bullet \mathrm{CH}_{3}---\mathrm{CH}_{2}=\mathrm{CH}_{2}$ & $\mathrm{CH}_{3}-\mathrm{CH}_{2}-\mathrm{CH}_{2} \cdot$ \\
\hline B3-LYP/6-311+G(d,p) & -39.855149 & -78.615380 & -118.461283 & -118.509149 \\
B3-LYP/6-311+G(3df,2p) & -39.857723 & -78.620901 & -118.469097 & -118.516379 \\
RB3-LYP/6-311+G(d,p) & -39.853763 & -78.615380 & -118.457609 & -118.507699 \\
RB3-LYP/6-311+G(3df,2p) & -39.856123 & -78.620901 & -118.465256 & -118.514738 \\
RMP2/6311+G(d,p) & -39.708635 & -78.346247 & -118.040905 & -118.099848 \\
RMP2/6311+G(3df,2p) & -39.731651 & -78.393176 & -118.111741 & -118.169390 \\
MPW1K/6-311+G(d,p) & -39.841551 & -78.583180 & -118.415962 & -118.475730 \\
MPW1K/6-311+G(3df,2p) & -39.843946 & -78.588329 & -118.423309 & -118.482528 \\
G3(MP2)-RAD & -39.785181 & -78.484212 & -118.258667 & -118.309597 \\
G3X-RAD & -39.824850 & -78.564963 & -118.379147 & -118.431623 \\
G3(MP2) & -39.775894 & -78.468625 & -118.232844 & -118.284700 \\
G3X & -39.822184 & -78.517460 & -118.305018 & -118.356895 \\
CBS-QB3 & -39.774012 & -78.466884 & -118.232702 & -118.283102 \\
CBS-QB3 (no spin correction) & -39.773899 & -78.466884 & -118.230267 & -118.282976 \\
W1h & -39.843504 & -78.604307 & -118.437319 & -118.490600 \\
W1//CCSD(T)/6-311G(d,p) & -39.843494 & -78.604526 & -118.437548 & --
\end{tabular}


TABLE S3: Total Energies (Hartrees) for QCISD/6-31G(d) Geometries, ZPVE Not Included

\begin{tabular}{|c|c|c|c|}
\hline Level of Theory & $\mathrm{CH} \equiv \mathrm{CH}$ & $\cdot \mathrm{CH}_{3}---\mathrm{CH} \equiv \mathrm{CH}$ & $\mathrm{CH}_{3}-\mathrm{CH}=\mathrm{CH} \bullet($ trans $)$ \\
\hline B3-LYP/6-311+G(d,p) & -77.356264 & -117.199925 & -117.258144 \\
\hline B3-LYP/6-311+G(3df,2p) & -77.361422 & -117.207627 & -117.265811 \\
\hline RB3-LYP/6-311+G(d,p) & -77.356264 & -117.196484 & -117.256716 \\
\hline RB3-LYP/6-311+G(3df,2p) & -77.361422 & -117.204006 & -117.264231 \\
\hline $\mathrm{RMP} 2 / 6311+\mathrm{G}(\mathrm{d}, \mathrm{p})$ & -77.113186 & -116.805972 & -116.863987 \\
\hline $\mathrm{RMP} 2 / 6311+\mathrm{G}(3 \mathrm{df}, 2 \mathrm{p})$ & -77.156851 & -116.873618 & -116.931230 \\
\hline MPW1K/6-311+G(d,p) & -77.317389 & -117.146581 & -117.217247 \\
\hline MPW1K/6-311+G(3df,2p) & -77.322511 & -117.154101 & -117.224566 \\
\hline G3(MP2)-RAD & -77.228434 & -117.000228 & -117.059652 \\
\hline G3X-RAD & -77.310548 & -117.121830 & -117.181909 \\
\hline G3(MP2) & -77.215670 & -116.978169 & -117.037794 \\
\hline G3X & -77.304277 & -117.113120 & -117.172946 \\
\hline CBS-QB3 & -77.214183 & -116.976863 & -117.036123 \\
\hline CBS-QB3 (no spin correction) & -77.214183 & -116.973604 & -117.034180 \\
\hline W1h & -77.351396 & -117.181197 & -117.242110 \\
\hline $\mathrm{W} 1 / / \mathrm{CCSD}(\mathrm{T}) / 6-311 \mathrm{G}(\mathrm{d}, \mathrm{p})$ & -77.351999 & -117.181708 & -117.242328 \\
\hline
\end{tabular}


TABLE S4: Total Energies (Hartrees) for QCISD/6-31G(d) Geometries, ZPVE Not Included

\begin{tabular}{lccc}
\hline Level of Theory & $\mathrm{CH}_{2}=\mathrm{CH}-\mathrm{CH}_{3}$ & $\bullet \mathrm{CH}_{3}--\mathrm{CH}_{2}=\mathrm{CH}-\mathrm{CH}_{3}$ & $\mathrm{CH}_{3}-\mathrm{CH}_{2}-\mathrm{CH}\left(\mathrm{CH}_{3}\right)^{\bullet}$ \\
\hline B3-LYP/6-311+G(d,p) & -117.9454320 & -157.7911309 & -157.8394252 \\
B3-LYP/6-311+G(3df,2p) & -117.9533732 & -157.8013159 & -157.8490737 \\
RB3-LYP/6-311+G(d,p) & -117.9454320 & -157.7875423 & -157.8379526 \\
RB3-LYP/6-311+G(3df,2p) & -117.9533732 & -157.7975609 & -157.8474225 \\
RMP2/6311+G(d,p) & -117.5461724 & -157.2416825 & -157.2989632 \\
RMP2/6311+G(3df,2p) & -117.6165884 & -157.3358001 & -157.3921223 \\
MPW1K/6-311+G(d,p) & -117.9038317 & -157.7362420 & -157.7962023 \\
MPW1K/6-311+G(3df,2p) & -117.9112920 & -157.7458281 & -157.805267 \\
G3(MP2)-RAD & -117.749920 & -157.524632 & -157.575370 \\
G3X-RAD & -117.871916 & -157.686388 & -157.738685 \\
G3(MP2) & -117.726516 & -157.490989 & -157.542740 \\
G3X & -117.858303 & -157.668491 & -157.720213 \\
CBS-QB3 & -117.724650 & -157.490907 & -157.541425 \\
CBS-QB3 (no spin correction) & -117.724650 & -157.488512 & -157.541291 \\
W1h & -117.931258 & -157.7644760 & -157.8177263
\end{tabular}


TABLE S5: Total Energies (Hartrees) for QCISD/6-31G(d) Geometries, ZPVE Not Included

\begin{tabular}{lccc}
\hline Level of Theory & $\mathrm{CH} \equiv{\mathrm{C}-\mathrm{CH}_{3}}^{-}$ & $\cdot \mathrm{CH}_{3}--\mathrm{CH} \equiv \mathrm{C}-\mathrm{CH}_{3}$ & $\mathrm{CH}_{3}-\mathrm{CH}=\mathrm{C}\left(\mathrm{CH}_{3}\right) \bullet(\operatorname{trans})$ \\
\hline B3-LYP/6-311+G(d,p) & -116.692415 & -156.535968 & -156.593673 \\
B3-LYP/6-311+G(3df,2p) & -116.700328 & -156.546350 & -156.603859 \\
RB3-LYP/6-311+G(d,p) & -116.692415 & -156.532658 & -156.592243 \\
RB3-LYP/6-311+G(3df,2p) & -116.700328 & -156.542860 & -156.602284 \\
RMP2/6311+G(d,p) & -116.316490 & -156.010146 & -156.067833 \\
RMP2/6311+G(3df,2p) & -116.384684 & -156.102161 & -156.158921 \\
MPW1K/6-311+G(d,p) & -116.644243 & -156.473076 & -156.542831 \\
MPW1K/6-311+G(3df,2p) & -116.651962 & -156.483111 & -156.552528 \\
G3(MP2)-RAD & -116.498607 & -156.270650 & -156.329596 \\
G3X-RAD & -116.622300 & -156.433785 & -156.493299 \\
G3(MP2) & -116.478031 & -156.240754 & -156.300030 \\
G3X & -116.609024 & -156.416690 & -156.476179 \\
CBS-QB3 & -116.476491 & -156.239610 & -156.298710 \\
CBS-QB3 (no spin correction) & -116.47649 & -156.23670 & -156.29697 \\
W1h & -116.682523 & -156.512655 & -156.573397
\end{tabular}


TABLE S6: Calculated Total Entropies $\left(\mathrm{J} \mathrm{mol}^{-1} \mathrm{~K}^{-1}\right)$ Using a Hindered Rotor Treatment for Low Frequency Torsional Modes

Level of Theory

HF/6-31G(d) B3-LYP/6-31G(d) B3-LYP/6-311+G(3df,2p) MPW1K/6-311+G(d,p) MP2/6-31G(d) QCISD/6-31G(d) CCSD(T)/6-311G(d,p)

\begin{tabular}{|c|c|c|c|c|c|c|c|}
\hline$\cdot \mathrm{CH}_{3}$ & 198.4 & 195.4 & 194.4 & 194.7 & 195.7 & 195.5 & 195.7 \\
\hline $\mathrm{CH}_{2}=\mathrm{CH}_{2}$ & 219.0 & 218.9 & 218.9 & 219.6 & 218.5 & 218.9 & 219.4 \\
\hline - $\mathrm{CH}_{3}---\mathrm{CH}_{2}=\mathrm{CH}_{2}$ & 295.6 & 296.1 & 295.7 & 296.8 & 290.0 & 292.6 & 294.0 \\
\hline $\mathrm{CH}_{3}-\mathrm{CH}_{2}-\mathrm{CH}_{2} \bullet$ & 296.3 & 295.9 & 296.1 & 295.9 & 293.5 & 295.0 & \\
\hline $\mathrm{CH} \equiv \mathrm{CH}$ & 198.9 & 201.6 & 200.0 & 200.8 & 205.3 & 204.4 & 202.1 \\
\hline$\cdot \mathrm{CH}_{3}---\mathrm{CH} \equiv \mathrm{CH}$ & 296.1 & 292.3 & 291.2 & 291.8 & 282.1 & 288.7 & 289.9 \\
\hline $\mathrm{CH}_{3}-\mathrm{CH}=\mathrm{CH} \bullet($ trans $)$ & 272.2 & 270.9 & 271.6 & 271.4 & 267.6 & 270.6 & \\
\hline $\mathrm{CH}_{2}=\mathrm{CH}-\mathrm{CH}_{3}$ & & 265.7 & & & & 265.9 & \\
\hline - $\mathrm{CH}_{3}---\mathrm{CH}_{2}=\mathrm{CH}-\mathrm{CH}_{3}$ & & 329.7 & & & & 329.7 & \\
\hline $\mathrm{CH}_{3}-\mathrm{CH}_{2}-\mathrm{CH}\left(\mathrm{CH}_{3}\right) \bullet$ & & 332.6 & & & & 331.2 & \\
\hline $\mathrm{CH} \equiv \mathrm{C}-\mathrm{CH}_{3}$ & & 256.8 & & & & 261.1 & \\
\hline$\cdot \mathrm{CH}_{3}---\mathrm{CH} \equiv \mathrm{CCH}_{3}$ & & 333.4 & & & & 330.3 & \\
\hline $\mathrm{CH}_{3}-\mathrm{CH}=\mathrm{C}\left(\mathrm{CH}_{3}\right) \bullet($ trans $)$ & & 310.2 & & & & 309.7 & \\
\hline
\end{tabular}


TABLE S7. Calculated Values of Forward $\left(\log \mathrm{k} /\left(\mathrm{L} \mathrm{mol}^{-1} \mathrm{~s}^{-1}\right)\right)$ and Reverse $\left(\log \mathrm{k} /\left(\mathrm{s}^{-1}\right)\right)$ Rate Constants at $298 \mathrm{~K}$ for Methyl

Radical Addition to Ethene and Ethyne Obtained Using the Hindered Rotor Model ${ }^{\mathrm{a}}$

\begin{tabular}{|c|c|c|c|c|c|c|c|c|c|c|}
\hline \multirow{3}{*}{$\begin{array}{l}\text { Level of Theory for } \\
\text { Frequency Factor }^{a}\end{array}$} & \multicolumn{10}{|c|}{ Level of Theory for Barrier } \\
\hline & \multicolumn{2}{|c|}{$\mathrm{RMP} 2 / 6-311+\mathrm{G}(3 \mathrm{df}, 2 \mathrm{p})^{\mathrm{b}}$} & \multicolumn{2}{|c|}{ RB3LYP/6-311+G(3df,2p) ${ }^{\mathrm{b}}$} & \multicolumn{2}{|c|}{$\mathrm{G} 3(\mathrm{MP} 2)-\mathrm{RAD}^{\mathrm{b}}$} & \multicolumn{2}{|c|}{ G3X-RAD ${ }^{c}$} & \multicolumn{2}{|c|}{$\mathrm{W} 1 \mathrm{~h}^{\mathrm{c}}$} \\
\hline & $\mathrm{CH}_{2}=\mathrm{CH}_{2}$ & $\mathrm{CH} \equiv \mathrm{CH}$ & $\mathrm{CH}_{2}=\mathrm{CH}_{2}$ & $\mathrm{CH} \equiv \mathrm{CH}$ & $\mathrm{CH}_{2}=\mathrm{CH}_{2}$ & $\mathrm{CH} \equiv \mathrm{CH}$ & $\mathrm{CH}_{2}=\mathrm{CH}_{2}$ & $\mathrm{CH} \equiv \mathrm{CH}$ & $\mathrm{CH}_{2}=\mathrm{CH}_{2}$ & $\mathrm{CH} \equiv \mathrm{CH}$ \\
\hline \multicolumn{11}{|c|}{ Forward (addition) rate constants } \\
\hline B3-LYP/6-31G(d) & 1.96 & 2.21 & 2.11 & 2.24 & 2.54 & 2.46 & 2.40 & 2.04 & 2.48 & 1.97 \\
\hline B3-LYP/6-311+G(3df,2p) & 1.99 & 2.43 & 2.14 & 2.46 & 2.58 & 2.67 & 2.44 & 2.25 & 2.52 & 2.19 \\
\hline QCISD/6-31G(d) & 1.62 & 1.46 & 1.77 & 1.49 & 2.21 & 1.71 & 2.06 & 1.29 & 2.14 & 1.23 \\
\hline $\operatorname{CCSD}(\mathrm{T}) / 6-311 \mathrm{G}(\mathrm{d}, \mathrm{p})$ & 1.45 & 1.79 & 1.60 & 1.82 & 2.03 & 2.04 & 1.89 & 1.62 & 1.97 & 1.56 \\
\hline \multicolumn{11}{|c|}{ Reverse $(\beta$-scission) rate constants } \\
\hline B3-LYP/6-31G(d) & -11.31 & -9.24 & -7.99 & -10.94 & -8.76 & -10.47 & -9.62 & -11.11 & -9.99 & -11.49 \\
\hline B3-LYP/6-311+G(3df,2p) & -11.48 & -9.48 & -8.16 & -11.18 & -8.94 & -10.71 & -9.79 & -11.35 & -10.16 & -11.73 \\
\hline QCISD/6-31G(d) & -11.26 & -9.28 & -7.94 & -10.98 & -8.71 & -10.51 & -9.57 & -11.15 & -9.94 & -11.53 \\
\hline
\end{tabular}

${ }^{a}$ Using a hindered rotor treatment for the low frequency torsional modes. ${ }^{b} \mathrm{~B} 3-\mathrm{LYP} / 6-31 \mathrm{G}(\mathrm{d})$ geometry. ${ }^{c} \mathrm{QCISD} / 6-31 \mathrm{G}(\mathrm{d})$ geometry. 
TABLE S8. Calculated Values of Forward $\left(\log \mathrm{k} /\left(\mathrm{L} \mathrm{mol}^{-1} \mathrm{~s}^{-1}\right)\right)$ and Reverse $\left(\log \mathrm{k} /\left(\mathrm{s}^{-1}\right)\right)$ Rate Constants at $298 \mathrm{~K}$ for Methyl

Radical Addition to Ethene and Ethyne Obtained Using the Harmonic Oscillator Approximation ${ }^{\mathrm{a}}$

\begin{tabular}{|c|c|c|c|c|c|c|c|c|c|c|}
\hline \multirow{3}{*}{$\begin{array}{l}\text { Level of Theory for } \\
\text { Frequency Factor }^{a}\end{array}$} & \multicolumn{10}{|c|}{ Level of Theory for Barrier } \\
\hline & \multicolumn{2}{|c|}{$\mathrm{RMP} 2 / 6-311+\mathrm{G}(3 \mathrm{df}, 2 \mathrm{p})^{\mathrm{b}}$} & \multicolumn{2}{|c|}{ RB3LYP/6-311+G(3df,2p) ${ }^{\mathrm{b}}$} & \multicolumn{2}{|c|}{$\mathrm{G} 3(\mathrm{MP} 2)-\mathrm{RAD}^{\mathrm{b}}$} & \multicolumn{2}{|c|}{ G3X-RAD ${ }^{c}$} & \multicolumn{2}{|c|}{$\mathrm{W} 1 \mathrm{~h}^{\mathrm{c}}$} \\
\hline & $\mathrm{CH}_{2}=\mathrm{CH}_{2}$ & $\mathrm{CH} \equiv \mathrm{CH}$ & $\mathrm{CH}_{2}=\mathrm{CH}_{2}$ & $\mathrm{CH} \equiv \mathrm{CH}$ & $\mathrm{CH}_{2}=\mathrm{CH}_{2}$ & $\mathrm{CH} \equiv \mathrm{CH}$ & $\mathrm{CH}_{2}=\mathrm{CH}_{2}$ & $\mathrm{CH} \equiv \mathrm{CH}$ & $\mathrm{CH}_{2}=\mathrm{CH}_{2}$ & $\mathrm{CH} \equiv \mathrm{CH}$ \\
\hline \multicolumn{11}{|c|}{ Forward (addition) rate constants } \\
\hline B3-LYP/6-31G(d) & 1.93 & 2.41 & 2.08 & 2.44 & 2.51 & 2.65 & 2.37 & 2.23 & 2.45 & 2.17 \\
\hline B3-LYP/6-311+G(3df,2p) & 1.96 & 2.57 & 2.11 & 2.60 & 2.55 & 2.82 & 2.41 & 2.39 & 2.49 & 2.33 \\
\hline QCISD/6-31G(d) & 1.56 & 1.59 & 1.71 & 1.62 & 2.14 & 1.83 & 2.00 & 1.41 & 2.08 & 1.35 \\
\hline $\operatorname{CCSD}(\mathrm{T}) / 6-311 \mathrm{G}(\mathrm{d}, \mathrm{p})$ & 1.23 & 1.76 & 1.38 & 1.79 & 1.82 & 2.00 & 1.68 & 1.58 & 1.76 & 1.52 \\
\hline \multicolumn{11}{|c|}{ Reverse $(\beta$-scission) rate constants } \\
\hline B3-LYP/6-31G(d) & -10.80 & -9.11 & -7.49 & -10.70 & -8.26 & -10.23 & -9.11 & -10.87 & -9.48 & -11.25 \\
\hline B3-LYP/6-311+G(3df,2p) & -11.09 & -9.30 & -7.78 & -11.00 & -8.55 & -10.53 & -9.40 & -11.17 & -9.77 & -11.55 \\
\hline QCISD/6-31G(d) & -10.72 & -9.00 & -7.41 & -10.81 & -8.18 & -10.34 & -9.03 & -10.98 & -9.40 & -11.36 \\
\hline
\end{tabular}

${ }^{a}$ Using a harmonic oscillator treatment for the low frequency torsional modes. ${ }^{b} \mathrm{~B} 3-\mathrm{LYP} / 6-31 \mathrm{G}(\mathrm{d})$ geometry. ${ }^{c} \mathrm{QCISD} / 6-31 \mathrm{G}(\mathrm{d})$ geometry. 